NASA Technical Memorandum 87325

\title{
Re-examination of Cumulative Fatigue Damage Analysis-An Engineering Perspective
}

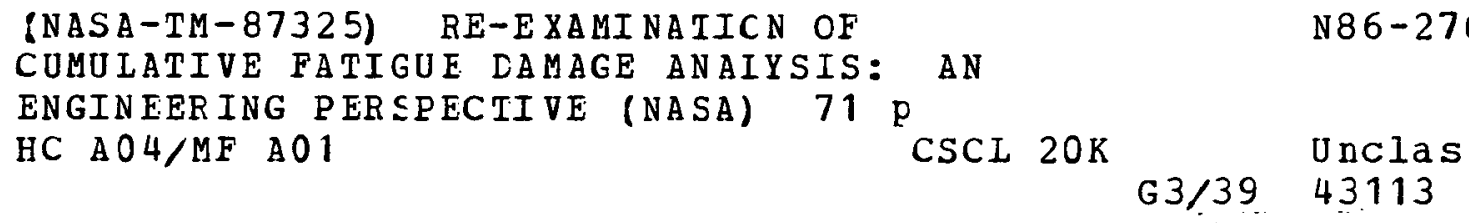

S.S. Manson

Case Western Reserve University

Cleveland, Ohio

and

G.R. Halford

Lewis Research Center

Cleveland, Ohio

Prepared for the

Symposium on Mechanics of Damage and Fatigue sponsored by the International Union of Theoretical and Applied Mechanics Haifa-Tel Aviv, Israel, July 1-5, 1985 
CONTENTS

Page

ABSTRACT . . . . . . . . . . . . . . . . . . . . . . . 1

INTRODUCTION . . . . . . . . . . . . . . . . . . . . . . . 1

CUMULATIVE FATIGUE FOR MECHANICAL LOADING . . . . . . . . . . . . . . 2

Fatigue Curve Convergence and Rotation . . . . . . . . . . . . . . 3

Basis ........................... 3

Critique.......................... . . . 5

Damage Curves. . . . . . . . . . . . . . . . . . . . . . . . 7

Basis for single-term damage curves . . . . . . . . . . . . . . 7

1. Flexibility of constants . . . . . . . . . . . . . . . . 8

2. Significance of the damage curves in relation to two-level

loadings........................ . . 9

Basis for a new double-term damage curve system . . . . . . . . . . 10

Double Linear Damage Rules. . . . . . . . . . . . . . . . . 11

Background . . . . . . . . . . . . . . . . . . . . . . . 11

Definition of linear and double-linear damage rules . . . . . . . . 12

Extension to multiple loading levels . . . . . . . . . . . . . . 14

Development of Practical Double-Linear Damage Rules . . . . . . . . . 16

Earlier work........................... 16

Recent development ..................... . . . 17

Application to multiple loadings . : . . . . . . . . . . . . . . 19

1. Multiple changes in loading involving two load levels . . . . . 19

2. Loadings involving three load levels............... 21

3. Cases involving large number of loading levels.......... 22

Construction of damage curves . . . . . . . . . . . . . . . . 22

Choice of the reference damage inines.............. . 24

4. Complex service cycle . . . . . . . . . . . . . . . . . . 25

DISCUSSION . . . . . . . . . . . . . . . . . . . . . . . . . . . 27

Restatement of Damage Curve Approach and Relation to DLDR . . . . . . 28

Modifying Procedure for Including Mean Stress Effects . . . . . . . . . 30

CAUTIONS ............................. . . . . . . . . . . 30

Effect of Stress Multiaxiality . . . . . . . . . . . . . . . . . . . . 31

Deformation systems in complex loading not revealed in individual

loadings . . . . . . . . . . . . . . . . . . . . . 31

Residual Stresses ......................... 32

Metallurgical Instabilities .................... . . . 32

CONCLUDING REMARKS . . . . . . . . . . . . . . . . . . . . . 35

APPENDIX A - DERIVATION OF THE DOUBLE DAMAGE CURVE APPROACH (DOCA) . . . 39

APPENDIX B - MODIFIED PROCEDURE FOR MEAN STRESS EFFECTS . . . . . . . . . 41 


\title{
RE-EXAMINATION OF CUMULATIVE FATIGUE DAMAGE ANALYSIS
}

- AN ENGINEERING PERSPECTIVE

\author{
S.S. Manson \\ Case Western Reserve University \\ Cleveland, Ohio 44706 \\ and \\ G.R. Halford \\ National Aeronautics and Space Administration \\ Lewis Research Center \\ Cleveland, Ohto 44135
}

\begin{abstract}
A method which has evolved in our laboratories for the past $20 \mathrm{yr}$ is re-examined with the intent of improving its accuracy and simplicity of application to engineering problems. Several modifications are introduced both to the analytical formulation of the Damage Curve Approach, and to the procedure for modifying this approach to achieve a Double Linear Damage Rule formulation which immensely simplifies the calculation. Improvements are also introduced in the treatment of mean stress for determining fatigue life of the individual events that enter into a complex loading history. While the procedure is completely consistent with the results of numerous two-level tests that have been conducted on many materials, it is st11l necessary to verify applicablitty to complex loading histories. Caution is expressed that certain phenomena can also influence the applicability - for example, unusual deformation and fracture modes inherent in complex loading - especially if stresses are multiaxial. Residual stresses at crack tips, and metallurgical factors are also important in creating departures from the cumulative damage theorles; examples of departures are provided.

INTRODUCTION

Treatment of accumulated fatigue damage has received a large amount of attention in recent years. The subject has been popular since palmgren [1]
\end{abstract}


first suggested what is now known as a "Linear Damage Rule." The same rule was later independently. proposed by Langer [2] and Miner [3]. However, it was soon recognized that while the method has merit for simple treatment of complex loading history, its predictions are often unconservative. Many alternative methods of analysis to predict behavior more accurately followed and it became necessary periodically to prepare review papers placing all the new methods into perspective, among them the ones by Newmark [4] Kaeche 1e [5], Manson [6], O'Ne111 [7] and Schive [8]. Schive's study lists neariy 200 references pertinent to the treatment of the problem and to the experimental programs conducted to evaluate the concepts.

No comprehensive report has appeared recently to review the considerable effort made in the past 12 yr since Schive's publication. Such a report is long overdue, and it was our original intent to prepare such a review. Because of space limitations, we found, however, that the review had to be limited. Thus, in the discussion that follows we shall restrict ourselves to elucidation and extension of those aspects of the subject with which we have had personal association. Although we will have occasion to refer to the work of others, these references will be brief. The more comprehensive review remains a subject for the future.

CUMULATIVE FATIGUE FOR MECHANICAL LOADING

Most theories of cumulative damage are based on observation of behavior at a second loading once the material has undergone loading, short of failure, at a previous level. This behavior is then extended by analogy to treatment of a complete history involving numerous loading levels. In our work we have used three basic concepts for the formulation of procedures: the effect of prior loading on the convergence and rotation of the fatigue curve in subsequent loading, the Damage Curve Approach (DCA), and the Double Linear Damage Rule (DLDR) concept. A critique of these methods will now be discussed. 


\section{Fatigue Curve Convergence and Rotation}

Basis. - Our entry into the cumulative fatigue damage field arose because of observations of a geometric relation between fatigue curves representing different degrees of damage either by prior fatigue or by damaging forms of machining. Bennett [9] in 1946 published the results of fatigue tests on specimens stressed at two successive levels, in Fig. 1. Line PA is the original S-N curve. Specimens prestressed at \pm 54 ksi for 33 percent of mean Iife, and then tested at other stress levels, yielded the new S-N curve PB. When 90 percent of the life was applied at $\pm 54 \mathrm{ksi}$, the remaining $\mathrm{S}-\mathrm{N}$ curve was. PC. It appeared to us that all three S-N curves projected to a single point $P$ at $\pm 60 \mathrm{ks} 1$ at 20000 cycles to fallure, and that the concept of convergence of $S-N$ curves contained the potential for the formulation of a cumulative fatigue damage procedure.

Another set of curves that suggested to us the convergence concept for cumulative fatigue is shown in Fig. $2[10]$. Here fatigue for a medium strength steel are shown for different types of surface finish. As, one proceeds from a ground finish through progressively rougher surface finishes, the S-N curves simply rotate about a point $P$. If we regard each form of machining as the imposition of a different level of surface damage similar to that which occurs in fatigue, this figure also suggests that each level of fatigue damage reflects its physical effect by causing a rotation of the $S-N$ curve for such materials in which the $S-N$ curve can be linearized.

On the basis of these observations, we proposed an approach to treatment of cumulative fatigue damage based on $S-N$ curve rotation [11]. A considerable test program was undertaken to check the validity of the approach, the major results being shown in Fig. 3 . Here, the first loading was at $\pm 100 \mathrm{ksi}$, for which the median life was determined to be 22000 cycles. A series of specimens were therefore subjected to various fractions of this 
$11 \mathrm{fe}$, and then the $\mathrm{S}-\mathrm{N}$ curve of the prestressed material was determined for each condition of prestress. These $\mathrm{S}-\mathrm{N}$ curves indeed appeared to be derivable by rotation of the basic $S-N$ curve of the material. Since one point on the "remaining" S-N curve is known by subtraction of the prestress cycles from the life at the prestress level, the predicted S-N curve can be determined by passing a straight line through this point from the point of rotation, $P$. While this point is unknown at the outset, it could be determined as a material constant, as in Fig. 4 , or it could be estimated by using the point on the $S-N$ curve at a stress near the uitimate tensile strength. Once the rotation point is known the effect of any loading sequence can be determined by successive application of the concept for treating two loadings. Each loading uses as its $S-N$ curve the rotated value already produced by the prior loadings, as illustrated in Fig. 4.

In Refs. [11] and [12], we assumed that each loading progressively reduced the endurance 1 imit. However, for brevity, we shall not review this issue. In many cases the existence of an endurance limit, and its progressive changes, is not of importance; however, even if endurance limits need to be considered, the rotation concept may not be the best vehicle for doing so. As we shall see later, we feel that the convergence framework has other limitations as well. Thus we prefer to devote more research to the other methods. Interested readers may, however, find Refs. [10] and [11] useful if there is need for information on changes of endurance limit.

In a more recent report Hashin and Rotem [13] have presented an interesting discussion of the convergence concept as a special case of a framework they have devised for cumulative damage analysis. They avoid the issue of determining the convergence point as a material constant, and take this point at a quarter of a cycle. They also speculate on alternative formulations, for example, when the convergence point is at the endurance 
1imit, Fig. 5. Such an approach would not, of course, properiy represent the usually observed loading order effect (i.e. high stress followed by low stress producing a cycle ratio summation less than unity, while a low-high sequence produces a sum greater than unity), but it is an interesting possibility for academic study. Hashin and Rotem also consider the case when stress versus cyclic life is linear on log-log coordinates, rather than semilogarithmic coordinates of figs. 3 and 4 . Of course, in this case the constant damage curves are presumed straight on the log-log coordinate system. Hashin and Rotem do not really require linearity in any particular coordinate system, only that the constant damage curves emanating from the focal point be a systematic set, never again intersecting anywhere. However, if they are not linear, then some other criterion must be introduced to establish the nature of the curvature.

Critique. - Although our initial efforts at implementing the convergence concept were quite vigorous, several limitations of the concept soon led us to seek alternate approaches. One was the obvious contradiction that would occur If the prior cycling produced a "remaining life" less than that at the convergence point $P$. For example, in Fig. 6 , if a prior loading at the stress level of point $A$ were applied to a degree that the remaining life were less than 1000 cycles, such as $B$, then the damage line after the initial cycling would have a positive slope, BP, which means that higher stress would produce longer life - a clear adabsurdum.

Secondly, as we conducted more tests on other materials it became clear that the convergence approach does not always properly represent the damaging effect of prior loading on subsequent loading at lower stress levels. We can 1llustrate this effect by a simple example in Fig. 7. Suppose, for example, we apply the initial loading at the stress level of point A. If we apply $x$ percent of the life, at this level, the remaining life, $(1-x)$ percent at 
this stress level is shown at point $A^{\prime}$. If, however, later testing is at stress level $B$, fallure would be expected at $B^{\prime}$, the linear projection of PA' to the stress level of $B$. Experience has indicated such a test would produce a lower life, at B", and in some cases a higher life, as at B"'.

To fllustrate the somewhat general expectation we resort here to the use of the Double Linear Damage Rule which we shall discuss later in the report. It is appropriate to use this rule for fllustration because it reflects more accurately the experience we have had with many materials. Figure 8 shows the results of detalled computations. Selected life fractions are assumed to be applied at stress level $A$, and the remaining $S-N$ curves are calculated by the DLOR approach to be $A^{\prime} B^{\prime}, A^{\prime \prime} B^{\prime \prime}$, etc. Two interesting results are evident: The curves $A^{\prime} B^{\prime}, A^{\prime \prime} B^{\prime \prime}$, etc. are not straight ines, as required by the linear-convergence concept; rather they are curved. Also, while $A^{\prime} B^{\prime}$ almost converges to $P$, the curves representing more highly fatigued material do not converge. After some fraction of preloading, they start to displace somewhat parallel to $A B$. Hence, the overall effect is one of rotation plus translation.

Incidentally, the rotation plus translation overcomes the difficulty envisioned earlier since the point $B$ can be displaced to any desired life level by prior loading without requiring the self-contradictory remaining life curve such as $B P$ in Fig. 6. This concept of rotation plus translation was first discussed by us in Ref. [14] when we introduced the OLDR concept. We have refined the approach in later publications, as we shall discuss, but the basic feature of rotation plus translation is inherent in all the variants we have studied.

On the basis of the above limitations of the rotation concept of convergence, we have not pursued this .concept further. The alternatives not 
only give more accurate behavior representation, but are easier to implement as we.11.

\section{Damage Curves}

Basis for single-term damage curves. - The use of damage curves to reflect the interaction of loadings at different stress or strain levels has been used since the concepts of cumulative fatigue damages have first been considered. In 1948 Richart and Newmark [15] introduced the idea, but did not provide definitive formulae for representing the damage curves in such a way as to predict quantitatively the high-low load sequences. Many attempts have been made since then to provide suitable analytical relations. In 1981 we made our own attempt [16] based on a large amount of experimental data for two-level loading conducted in our laboratory. The form we chose,

$$
D=\left[\frac{1}{0.18}\right]\left[a_{0}+\left(0.18-a_{0}\right)\left(\frac{n}{N}\right)^{\frac{2}{3}(N)^{0.4}}\right]
$$

was based on analogy to early crack growth. Figure 9 shows the concept as we applied it. The curves represent the damage accumulation for each of the decade life levels from $10^{2}$ to $10^{6}$. As an example, if $n_{1}$ cycles are applied at the $10^{3}$ cycles to fallure level the damage accumulated will be represented by point $A$. Changing to a second load level, which produces a ilfe of $10^{5}$ cycles to fallure, moves us to point $B$ at the same damage as $A$, and cycling can continue for $n_{2}$ cycles as damage accumulates from $B$ to fallure at $C$. Thus, if the damage curve formulae are known, it is easy to calculate $n_{2} / N_{2}$ once the $n_{1} / N_{1}$ is specifted if $N_{1}$ and $N_{2}$ are known. The subscripts refer to sequence of the applied loadings, 1 is the first and 2 is the second level. A more convenient type of plot is shown in Fig. 10 where the life scale is normalized to fallure life. From this figure it is clear that if the first loading is at the higher stress (lower life) and the second is at the lower stress (higher life) the sum of the cycle ratios will be 
smaller than unity. Since this is the most commonly observed behavior, the qualitative nature of the damage curves is correct. Before discussing the precise quantitative implications it is useful to clarify a few points.

1. Flexibility of constants. - If we express the relation that the damage at $A$ and $B$ are equal in Fig. 10, i.e., $D_{A}=D_{E}$, then it is clear that the $a_{0}, 0.18$ and $2 / 3$ terms in Eq. (1) cancel, and that

$$
\frac{n_{2}}{10^{5}}=1-\left[\frac{n_{1}}{10^{3}}\right]^{\left[\frac{10^{3}}{10^{5}}\right]^{0.4}}
$$

More generally for any combination of life levels $N_{1}$ and $N_{2}$ we obtain the Damage Curve Approach (DCA).

$$
\left.\frac{n_{2}}{N_{2}}=1-\left[\frac{n_{1}}{N_{1}}\right]^{\left[\frac{N_{2}}{N_{2}}\right.}\right]^{0.4}
$$

Thus the relation between $n_{2} / N_{2}$ and $n_{1} / N_{1}$ depends only on the ratio $\mathrm{N}_{1} / \mathrm{N}_{2}$, and is independent of any constants that might be introduced in place of the $a_{0}, 0.18$, and $2 / 3$. Thus, suppose we replace $a_{0}$ by zero, the 0.18 by 1.0 and the $2 / 3$ by 0.02512 (equal to $\left[1 / 10^{4}\right]^{0.4}$ ), the damage curve relation for the $10^{4}$ life becomes linear and the damage curve for any other value of $N$ becomes simplified. In fact we can choose to linearize the damage curve for any $N_{\text {ref }}$ by replacing the $2 / 3$ by $\left[1 / N_{\text {ref }}\right]^{0.4}$. In this case

$$
D_{N_{\text {ref }}}=\frac{n}{N_{\text {ref }}}
$$

and 


$$
\left.D_{N}=\left[\frac{n}{N}\right]^{\left[\frac{N}{N_{\text {ref }}}\right.}\right]^{0.4}
$$

Figure $11(a)$ shows the damage curves for 11 fe levels from 10 to $10^{7}$ when the damage curve for $10^{4}$ is taken as the reference straight ine. More generally, these same curves are obtained when any life level $N_{2}$ is taken as the reference life. The damage curves for other lives are labeled as a function of $N / N_{\text {ref }}$, as seen in Figs. $11(a)$ and (b) which produce the same curves for equal values of $N / N_{\text {ref }}$ whether the reference life is taken as $10^{3}$ or $10^{4}$ cycles. Only one set of damage curves is thus needed as long as the designation is according to $N / N_{\text {ref }}$. Note that the reference life level can be used as the first, second, or any other number in a sequence of cumulative loadings.

\section{Significance of the damage curves in relation to two-level loadings. -} Figure 12(a) shows the damage curves for two-level tests, using the first (lower) life $N_{1}$ as the reference which is forced to be a straight line. If we wanted to use these curves to obtain the $n_{2} / N_{2}$ versus $n_{1} / N_{1}$ curves for such a test, the procedure would be to go from $A$ to $B$ to produce the arbitrary $n_{1} / N_{1}$, determine $C$ at the same damage as $B$, and measure $n_{2} / N_{2}$ as damage proceeds from $C$ to $E$. Thus $n_{2} / N_{2}$ is equal to length $C D$. If we plot $n_{2} / N_{2}$ versus $n_{1} / N_{1}$, as in Fig. 12(b), the point $C^{\prime}$ results. For other cholces of cycle ratio $n_{1} / N_{1}, n_{2} / N_{2}$ falls at other points along the curve $A^{\prime} C^{\prime} E^{\prime}$. Examination of Figs. 12(a) and (b) reveals that the curves ACE and $A^{\prime} C^{\prime} E^{\prime}$ are similar. One can be obtained from the other by a $90^{\circ}$ rotation about the central point $x$. We can then generalize that if the curves of Fig.s. $11(a)$ and (b) represent the damage line relationships, the expected $n_{1} / N_{1}$ 
versus $n_{1} / N_{2}$ curves can be obtained by a clockwise $90^{\circ}$ rotation. Such a family of curves is shown in Fig. 12(c).

Basis for a new double-term damage curve system. - Although the single-term damage curve system was chosen by us to agree with many experimental results generated in two level tests, there is one feature of the system that has been of some concern to us from the very beginning of its use. This feature can be seen in Fig. 12(c). The initial drop of $n_{2} / N_{2}$ for a small value of $n_{1} / N_{1}$ is exstremely rapid. Since the choice of the damage curve relation was derived mostly for two-level tests which did not involve such low values of $n_{1} / N_{1}$ as to be within the troublesome. low range, the choice was not in conflict with our experiments. However, after we recognized that the resulting feature of the damage curve analysis was probably unrealistic, we decided to attempt to improve its characteristics. Brief attempts to modify the terms in Eq. (1) indicated that it would be difficult to improve the very low $n_{1} / N_{1}$ part of the curve while retaining its good features in the remainder of the range. We decided, therefore, that a more appropriate way would be to add a term that would have a large significance at low values of $n_{1} / N_{1}$, but only relatively small effect for larger values. In our procedure to alter the Damage Curve equation we were guided by our Double-Linear Damage Rule analysis [16] which will be discussed later.

To distinguish the new double-term damage curve from the original single-term expression, we refer to it as the Double-Damage Curve Approach (DDCA). Details of the derivation are given in Appendix $A$ and only the final results are presented here:

$$
D=\left[\frac{n}{N_{f}}\right]\left\{a_{1}^{\gamma}+\left[1-q_{1}^{\gamma}\right]\left[\frac{n}{N_{f}}\right]^{\gamma\left(q_{2}-1\right)}\right\}^{1 / \gamma}
$$

where 


$$
q_{1}=\frac{0.35\left[\frac{N \text { ref }}{N}\right]^{\alpha}}{1-0.65\left[\frac{N_{\text {ref }}}{N}\right]^{\alpha}} ; \quad q_{2}=\left[\frac{N}{N_{\text {ref }}}\right]^{\beta} ; \quad \alpha=0.25, \beta=0.4, Y=5
$$

Note that $q_{1}$ is the slope of the first straight line segment of the 0 versus $n / N$ curve in the OLDR formulation, discussed in the next section. The $\mathrm{q}_{2}$ term is the exponent of the $D$ versus $n / N$ curve in the DCA. formulation, Eq. (5), and the constant $Y$ is introduced to represent two intersecting straight lines by a curve having a single methematical formula.

Direct comparison of the newly formulated DOCA, Eq. (6), with the DLOR and the original DCA is given in Fig. 13 for values of $\mathrm{N}_{\text {ref }} / \mathrm{N}$ of $10^{-1}$, $10^{-2}$, and $10^{-3}$. As expected, the DDCA equation conforms closely to the DLDR in the early portion of the Phase I regime but blends directly into the DCA curves which are also close to the DLDR in Phase II.

\section{Double Linear Damage Rules}

Background. - One concept that developed early to explain deviations for the linear damage rules was that fatigue was at least a two-stage process crack initiation and crack growth; if the two processes developed at different rates for different life levels, there is no reason for expectation of a linear damage accumulation rate for all life levels. An immediate conjecture was that while it is still possible for linear damage accumulation to develop during the initiation stage, and linear accumulation during the propagation stage, propagation might develop at different fractions of life for the different life levels. Therefore, a mixture of loadings involving several life levels would not be amenable to analysis by a single linear damage analysis. Thus two damage rules were needed; one for initiation and one for propagation. 
Probably the first to suggest analysis by two linear damage rules was Langer [2]. Later, Grover [19] again proposed the concept independently. However, neither proposed quantitative relations for establishing when one process ended and the other began, nor how to treat problems involving more than one loading level. Manson [14] made an early attempt in 1966 on the basis of limited data, but the relation proposed at that time did not prove to be sufficiently accurate to predict results of later experiments, so additional attempts [20] were made. Even these attempts were not sufficiently general, or of sufficient defilltive procedure to be of general engineering use. However, in 1981 Manson and Halford [16] refined the formulation and procedure to be directly applicable to the analysis of engineering problems. Additional valuable contributions both to formulation, and possibly to defining the physical mechanisms involved, were made by Miller and his co-workers [21 and 22].

Definttion of linear and double-linear damage rules. - Rather than progressing from damage curve to damage curve as loading increments accumulate, considerable simplification can be introduced by using the well known concepts of single and double-linear damage rules. Consider, for example, Fig. 14(a), which involves two loading life levels. If the damage curves are linear, it is possible to normalize each by dividing by the fallure life, reducing both damage lines to a single line. Obvtously, for this case the procedure discussed for summing damage increments leads to $\Sigma \mathrm{n} / \mathrm{N}_{\mathrm{f}}=$ 1, i.e., the linear damage rule (LDR). Adding loading levels to the history does not change this conclusion as long as each damage level is straight. Even if the damage curves consist of broken straight line segments, as shown in Fig. 14(b), a linear damage rule will apply if normalizing relative to fallure life reduces all damage lines to a single set of broken lines. or, the damage lines are curvilinear over their entire range, as shown 
in Fig. $14(\mathrm{C})$, a linear damage rule will apply if all damage lines are reduced by normalization to a single curve. Nor does it matter if more loading levels are involved, as long as all reduce to a single curve by one normalization. The merit of treating damage accumulation by a linear damage rule rather than by damage curves is great simplification; loading order becomes unimportant, and we sum all the cycle ratios without regard to sequence, even when individual loadings at one level are interspersed with loadings at the other. The amount of calculation involved, and the associated bookkeeping of damage accumulation is, therefore, drastically reduced.

Now consider the case shown in Fig. 15(a). Two damage curves are shown: one is straight, the other shows a single break. Normalizing by dividing by fallure life produces the triangular shape in Fig. 15(b). Let us label as $D_{I}$ the region of damage from the origin to the level of $A B$, and designate it as Phase $I$ since the linear nature of $O A$ and $O B$ suggests that in this region of damage accumulation the damage curves have something in common. Similarly the Phase II region from $A B$ to $C E$ can be labeled $D_{I I}$. If in the $D_{I}$ region we divide all cycle levels of curve $O A$ by $N_{A}$ and all damage levels by $D_{I}$, the line $O A$ " of Fig. 15(C) results. The same straight line results when we normalize $O B$ by division by $N_{B}$ and $D_{I}$.

Figure $15(\mathrm{C})$ now states that as long as the damage has not yet reached $D_{1}$, we can use a single linear damage rule. In this region, it does not matter how many times load level changes from $O A$ to $O B$, the cycle ratios along $O A$ can be summed independently, and those along $O B$ independently. When the sum of cycle ratios reaches unity. Phase $I$ damage $D_{I}$ has been completed.

Figure $15(d)$ is constructed in a simflar manner from the segments $A C$ and $B E$ of Fig. 15(a). Cycles are divided by $N_{A C}$ and $N_{B E}$, respectively, and the increase in damage beyond $D_{I}$ is divided by $D_{I I}$. 
Since both segments collapse to a single straight line, we can treat damage accumulation in this region as independent of application order as well. Thus, no matter how many times, or in what sequence, the loading changes from the $O A C$ to the $O B E$ level, the damage accumulation depends only on the total sum of cycle ratios at each level. But cycle ratios must of course be determined relative to the cycles required to apply the $D_{I I}$ damage, not total cycles.

In our early work we designated the DI region as "crack initiation" and the $D_{I I}$ region as crack propagation. However, we could detect no. cracks at the transition between the regions, and for other philosophical reasons discussed in [16] we have abandoned designations according to physical interpretations, and now simply call these regions I and II. As we shall later discuss, the engineering use of the concept is not restricted by absence of physical designation.

Extension to multiple loading levels. - In the foregoing discussion we have limited the consideration to two loads involving only one break point level in the damage relations. As long as breaks occur at the same 0 level, the procedure could be generalized to any number of load levels without change in final result. The general case involving more than two load levels requires that the break points be at various levels. Consider first Fig. 16(a) in which the damage line OFG has been added. We could, of course, divide the damage range into three regions: below $F$, between $F$ and $B$, and above B. This would lead to triple-linear damage curves, (Fig. 16(b)) and a triple-linear damage rule (Fig. 16(c)). Inclusion of more than three loading levels would involve even a larger number of distinct inear regions, and the desired simplicity is lost. We have, therefore, chosen an alternate approach. Only two regions are used, but they are, chosen so as to preserve fidelity to the important damage lines in a given loading 
sequence and sacrifice fidelity to those loadings which do not have an. important effect.

The basic philosophy is 11lustrated in Fig. 17. Here we choose OAP as the damage line for the life level $N_{1}$ of the loading which will cause the major damage in a particular application. As noted earlier, we can choose this damage line as a straight $45^{\circ}$ line, ending at point $P$ where $D=1.0$ and $n / N_{1}=1.0$. The line $O B P$ for a life $N_{2}$ is the normalized damage Iine for the loading level that is of next importance in producing damage for this loading history. (Before performing the analysis the values of $N_{1}$ and $\mathrm{N}_{2}$ may not be known, and an engineering judgment is made. The subsequent analysis provides better choices of $N_{1}$ and $N_{2}$, which quickly converge to appropriate values.) As long as only the life levels $N_{1}$ and $\mathrm{N}_{2}$ are involved in a loading sequence, we can proceed as already discussed. If another life level $N_{3}$ (between $N_{1}$ and $N_{2}$ ) is added, however, its damage curve will be OCP, with a breakpoint at a higher damage level, as previously discussed. One way to keep the breakpoints at the same level of damage, is to replace $O C P$ by $O C^{\prime} P$, choosing $C^{\prime}$ where $O C$ intersects the damage level $A B$. We then have a damage line which coincides with the real damage line in region $O C^{\prime}$ and lies above it in the region C'CP. The replacement damage line thus errs on the conservative side since we attribute a greater damage to an increment of loading at this level than would occur by using the real damage line. Similarly, if we need to consider a loading level with a life greater than $\mathrm{N}_{2}$ we could conservatively replace its damage curve ODP by OD'P. Once we have forced all breakpoints to occur at the same damage level we can reduce the problem to one of a double-linear damage rule. While the schema of $\mathrm{Fig} .17$ is a viable framework for reducing any problem to one involving a double-linear damage rule, the procedure we 
have actually adopted is easier to apply and gives very good results, as will be discussed.

Development of Practical Double-Linear Damage Rules

Earlier work. - Our objective for many years has been to establish a practical double linear damage rule framework usable in design and analysis. Progress has been gradual. Our first report dates back 20 yr when we first proposed [14] that "crack initiation" and "crack propagation" as the phases within which the two linear damage rules applied. Using limited information on one material it first suggested that the crack initiation period be $N_{f}-15 N_{f}^{0.6}$ whenever $N_{f}>750$ cycles, and equal to 0 for $N_{f}<750$. The propagation period would, of course, be $15_{f}^{0.6}$ and $N_{f}$, respectively. This rule was simple, but unfortunately not sufficiently accurate for other materials. In 1967 a second report [20] examined data on other materials and test conditions. We were forced to conclude that not only could we not, in general, use the simple formula $N_{p}=15 N_{f}^{0.6}$ for the propagation phase, but that it was inappropriate to use the terms "crack initiation" and "crack propagation" as descriptors of the two phases. Not only could we not detect any crack at the kneepoint of a two-level test, when the "crack" should have "initiated," but the kneepoint shifted as we combined different levels of the second loading with identical first load levels. For example, if a 1000 cycle life level loading is followed by a 10000 cycle life load level, the kneepoint implies that "crack initiation" starts at about 200 cycles. However, if the 1000 cycle life level is followed by a loading of 1 ife $10^{6}$ cycles, the implied "crack initiation" life for the 1000 cycle level now becomes only about 60 cycles. Since the material cannot sense while it is initially loaded at the 1000 cycle level what the subsequent loading will be, why should it "crack" 
after 60 cycles in one case and 200 cycles in the other? For other choices of loading levels even greater discrepancles could result. Our conclusion was that the type of linearity we perceived for so many materials and loading combinations was not a manifestation of "crack initiation" and "propagation," but of some other phenomenon that we did not yet fully understand.

In the 1967 report we salvaged the concept of a double-1inear damage rule, but changed the terminology, calling the regions where the two rules appeared to be valid as Phase I and Phase II. That we did not know the physical significance of these phases did not invalidate the potential use of the concept, but application required an empirical determination of the kneepoint between the two phases for each sequence of loading to be evaluated. Unfortunately, such an approach is not appealing to a designer. When one is trying to choose a material among many candidates, and when the loadings may in fact even depend on the material chosen, few designers have the inclination, the facilities, or the lead-time to conduct the required tests.

Recent development. - The matter lay dormant for many years, but interest was again.stimulated in this subject during our evaluation of the Damage Curve Approach (DCA) for treating cumulative fatigue damage. The model fashioned for DCA was, naturally, also based on the considerable amount of data developed for two-level tests on many materials, and the type of results that we obtained were as shown in Fig. 18. The continuous curves in this figure show the results for the damage curves used at that $t$ ime. We then question what these damage curves would look like if we replaced them with two

tAs already discussed, our use of damage curve equations using only a single term gave somewhat unrealistic results for low values of $n_{1} / N_{1}$. Use of the double damage curve equation (6) discussed earlier produces results very similar to the dotted lines in this figure. 
straight lines, and sketched the dotted lines shown in the figure. of special interest was that the damage curves for a sequence involving two load levels $N_{1}$ and $N_{2}$ depended only on the ratio $N_{7} / N_{2}$, and not on the individual values of $N_{1}$ and $N_{2}$. The same curve would be obtained if the first life level were 1000 cycles and the second 10000 cycles as would result when a 100000 cycle loading was followed by a $10^{6}$ cycle loading. therefore we would have to expect the same implication for the OLDR.

This observation was precisely the clue we needed to understand the confusing results derived by stating a DLDR based on crack initiation and crack propagation concepts. Referring to the illustration used earlier, if a 1000 life level loading were followed by a 10000 cycle level, the $N_{1} / N_{2}$ ratio is 0.1 , and we should expect the apparent knee to be at about 200 cycles. But if the 1000 cycle life loading level is followed by a $10^{6}$ life level, the $N_{1} / N_{2}$ ratio becomes $10^{-3}$, and we should expect the apparent knee to be at about 60 cycles. It was not a matter of "initiating" a crack at either of the two knee points. Rather, it was a question of which $\mathrm{N}_{7} / \mathrm{N}_{2}$ curve was involved. By defining Phase I as the region up to the kneepoint, and Phase II as the region beyond the kneepoint, we can avoid the philosophical question of its physical significance and yet devise a simple but useful analytical procedure.

We returned to our raw data to see if an equation could be derived for the coordinates of the kneepoint based on the parameter $N_{1} / N_{2}$ rather than on the $N_{1}$ parameter as we had previously sought. The results are shown in Fig. 10. Indeed the data of the kneepoint coordinates normalized better on the basis of the ratio $N_{1} / N_{2}$. (By comparison we show in Fig: 20 that when only $N_{1}$ was used to correlate the horizontal coordinate of the kneepoint, as required if crack initiation occurs here, the results are poor.) 
From Fig. 19 the coordinates of the kneepoint are

$$
\left.\left.\frac{n_{1}}{N_{1}}\right]_{\text {knee }}=0.35\left[\frac{N_{1}}{N_{2}}\right]^{\alpha} ; \frac{n_{2}}{N_{2}}\right]_{\text {knee }}=0.65\left[\frac{N_{1}}{N_{2}}\right]^{\alpha} ; \quad \alpha=0.25
$$

Once the kneepoint is established, it becomes possible to re-examine all the two-level tests we have conducted to determine if the data could have been predicted in advance. Figure 21 shows how double-linear relations, using Eq. (7) to establish the kneepoint, have worked for three materials - 300CVM, SAE4130, and T1-6Al-4V. The agreement generally is quite good.

Application to multiple loadings. - We next consider how to treat multiple loading levels.

1. Multiple changes in loading involving two load levels: Consider a case involving a mixture of loadings which if applied individually would produce 1000 and 100000 cycles to fallure. In this mixture changes occur from one load to the other many times in arbitrary. order. A simple case, for example, is one in which blocks consisting of 1 percent each of the basic loadings are successively applied until fallure occurs. Each block contains 10 cycles of the loading which alone produces a 1000 cycle life, and 1000 cycles of the loading which alone produces 100000 cycle life. We seek to determine how many blocks can be sustained. Figure 22 shows how this problem would be organized.

The first step is to reinterpret the DLDR in terms of equivalent damage curves. Figure $22(a)$ shows the double-linear representation on the basis that Phase I is consumed entirely at the $10^{3}$ cycle-to-fallure level, and Phase II at the $10^{5}$ cycle-to-fallure level. The coordinates of point $B$ are computed by Eq. (7), and the numerical values are shown in the lower, part of the figure. Figure 22 (b) shows the equivalent damage lines obtained by a $90^{\circ}$ counterclockwise rotation of $\mathrm{Fig} .22(\mathrm{a})$, as previously discussed. Thus $A^{\prime} B^{\prime \prime} C^{\prime}$ represents damage accumulation at the $10^{3}$ life loading, while 
$A^{\prime} B^{\prime} C$ shows how damage accumulates for the $10^{5}$ life loading for a problem which contains only these two loadings.

The next step is to track the damage accumulation as successive loading blocks are applied. In the early loading the damage accumulates along A'B" when the $10^{3}$ loading is applied, and along $A^{\prime} B^{\prime}$ when the $10^{5}$ loading is applied. Thus the straightforward way is to move between these two lines as block after block is applied, until the damage reaches the level $B^{\prime \prime} B^{\prime}$. Beyond this point the pertinent damage lines to use are $B^{\prime \prime} C^{\prime}$ and $B^{\prime} C^{\prime}$. Again the straightforward procedure is to move back and forth between these two lines, increasing damage until point $C$ is reached. Proceeding in this way requires many changes from one line to the next (over 52 times).

However, if we recognize that until the damage reaches $B^{\prime \prime} B^{\prime}$, both damage lines are straight, then a linear damage rule is valid and the loading order does not matter. We can then assume that the same effect will be produced by separately lumping together all the cycles of the $10^{3} 1$ ife level and all the cycles of the $10^{5}$ level. Thus if it requires $B_{1}$ blocks to complete Phase I, then $10 \mathrm{~B}$, cycles of the $10^{3}$ life level and $1000 \mathrm{~B}$. cycles of the $10^{5}$ life level will be applied. Since the number of cycles to complete Phase I for the $10^{3}$ 11fe level is 111 , and for the $10^{5}$ iffe level, is 79445 , the equation for $B_{1}$ in Fig. 22 applies, and it is seen that 9.74 blocks $^{+}$are required to complete Phase I. By similar reasoning, it follows from the calculations of $\mathrm{Fig} .22(\mathrm{~b})$ that 16.67 blocks are required to complete Phase II, for a total number of 26.41 blocks. This is in contrast to 50 blocks that would be unconservativeiy predicted by the Miner Linear Damage Rule (LOR).

tFor simplicity we accept decimal values of blocks, even though a nonintegral value means that some loadings of one level are required to complete the block without being matched by the other loading. 
The simplicity of this type of approach is evident for the elementary problem 1llustrated here. When extended to more complex loading histories the saving of calculation labor is even more striking.

2. Loadings involving three load levels: In Fig. 23 we have added a third load level which by itself would produce an intermediate iffe of 10000 cycles, and again assume block loadings involving 1 percent of individual lives. As seen in the figure, $B^{\prime}$ and $B^{\prime \prime}$. are obtained as before. The problem is with the damage curve that is to represent the $10^{4}$ cycle life level. Its kneepoint, based on individual combination with the $10^{3}$ cycle life loading is at $D$, which does not have the same vertical coordinate as $B^{\prime} B^{\prime \prime}$. To use a DLDR we must find a suitable replacement for the line ADC having a break-point along $B^{\prime} B^{\prime \prime}$. Several alternatives are possible. One is to choose $D^{\prime}$ at the average where $A D$ and $C D$ intersect the $B^{\prime} B^{\prime \prime}$ line so that $A D^{\prime} C$ replaces $A D C$. A second way is to use the interpolation formula shown in Fig. 23 which was derived in [16]. Basically, it is an analytical relation for the cycle ratio to the end of Phase I which is consistent with the formulas used to get the coordinates of $B$ ' and "B" and which both interpolates and extrapolates in a manner consistent with experimental experience. This matter will be discussed in connection with Fig. 24 .

For the case of the 10000 cycle life in Fig. 23, the formula produces a location for $D^{\prime}$ at $n / N_{f}=0.476$ which is nearly the same as averaging D" and D''. The replacement damage line $A D^{\prime} C$ in $F i g .23$ is seen to be below the real damage line $A D C$ in the Phase $I$ range, and above it in the Phase II range, compensating for errors introduced by forcing all breakpoints to have the same ordinate.

Once we have forced all the breakpoints to be at the same ordinate, linear damage rules can be expressed for the Phase I region below this ordinate, and for the Phase II region above it. Damage calculations are shown 
in Fig. 23 for the case of blocks of 10,100 and 1000 cycles applied respectively at $10^{3}, 10^{4}$, and $10^{5}$ iffe levels. As before, we first find out how much Phase I damage a single block of loading produces, and then determine how many blocks it takes to complete Phase I. Similarly we calculate how many blocks are required to complete Phase II, and therefore the total number of blocks to fallure. In this case, as seen in Fig. 23, a total of 20.7 blocks are required. The LOR would have predicted 33.3 blocks, about 60 percent higher than the DLDR computation. To treat this simple problem by the DCA would have required changing progressively from one to the other of the three damage curves, approximately 60 times. Even for this simple case the calculations would involve an order of magnitude more labor than the OLDR procedure. But the result would have been nearly identical to that obtained by the DLDR. For the DCA analysis the prediction is 21.0 blocks; for the DDCA the prediction is 23.3 blocks, and for the DLDR analysis it is 20.7 blocks.

3. Cases involving large number of loading levels: In the more complex problem involving many loading levels two important questions arise: 1) which two load levels should be used as the baseline values around which to establish when Phase I ends and Phase II begins, and 2) how to construct the damage curves for loading levels in the extrapolated range, that is lower than the lower life of the reference damage lines or higher than the higher life of the reference damage lines.

Construction of damage curves. - We address the second of these questions first by extending the simple problem we have been discussing using four loading levels shown in Fig. 24 , loadings which alone would produce lives of: $10^{3}, 10^{4}, 10^{5}$, and $10^{6}$ cycles. Also, to avoid the issue of which loading produces most of the damage, we assume that each block contains 1 percent of the fallure cycles for each loading level individually. Figure $24(a)$ shows how each of the damage lines would be drawn if the $10^{3}$ 
damage line is taken as the $45^{\circ}$ reference line. Note that the kneepoints do not have the same value, as follows from Eq. (7). One of these kneepoints needs to be chosen, thus allowing us to retain only two damage curves, but the other two must be compromised.

In Fig. 24(b) the kneepoint associated with the $10^{3}$ and $10^{6} 11 \mathrm{fe}$ levels is retained, and the $10^{4}$ and $10^{5}$ ilfe damage lines are compromised. They are constructed in the same manner as used in Fig. 23 , using the formula shown in this figure. The calculations for blocks to complete Phases I and II are then made in an identical manner as in Fig. 23, resulting in 11.47 blocks. In Fig. $24(\mathrm{c})$ the $10^{3}$ ilfe damage line is paired with the $10^{5}$ life damage curve, thus requiring the compromising of the $10^{4}$ and $10^{6}$ damage curves, - one in the interpolated range of the faithful damage curves, and one in the extrapolated range. Again the same formulas shown in Fig. 23 are used to establish the location of the compromised damage curves, and the procedure for determining number of blocks is the same. For this case the computed number of blocks is 12.03 .

Finally, in Fig. $24(\mathrm{~d})$, the $10^{3}$ and $10^{4}$ life damage lines are faithfully retained, and damage curves for $10^{5}$ and $10^{6} 11$ fe are compromised. Again, of course, the formulas of fig. 23 are used to establish the coordinates of the kneepoints, and the computation procedure is the same as in the previous cases. Half of the damage curves are now in the extrapolated range, but the computed number of 13.77 blocks is not far different from the other.

It should be recognized that this example represents an extreme case. Here the contribution of each loading to the damage accumulation is the same, since in each block all contribute equal numerical values of damage. Yet, even though equally damaging damage lines were compromised, the computed 
blocks differed by less than 10 percent. But the conclusion is clear that if the reference damage curves are at least as important in contributing damage as those damage lines that have been compromised, the use of our basic equation shown in Fig. 23 is adequate for establishing the damage ilines used in the analysis.

Choice of the reference damage lines. - It stands to reason that the degree of error introduced by compromising fidelity of a damage line depends on how importantly the loading associated with that damage line contributes to the total damage. Thus the most important loading events should be used as the reference uncompromised damage lines while the lesser events can be compromised without introducing much error. If, as seen in the example of Fig. 24, compromising important lines do not seriously affect the resulting calculations, then compromising the lesser contributors should not be too serious. But first we have to know which are the important events. It would seem that the answer could be obtained by a successive approximation approach. The first step could be an ordinary linear damage analysis, and the cycle ratio at fallure for each of the contribution events determined. The two most damaging events can be taken as $N_{1}$ and $N_{2}$, where $N_{1}$ is the lower of the two. If there is ambiguity because several events contribute approximately the same damage, then choose $N_{1}$ and $N_{2}$ as far apart as. possible, so that other important damaging events will have life values between them. Then make a DLDR analysis, and review which events are now the most damaging. If other loadings now appear to be better candidates as $\mathrm{N}_{1}$ and $N_{2}$, redo the analysis. Since each analysis is quite simple, consisting of only two sets of computations each of which is the same as a LOR analysis, this procedure should not be too demanding. Further refinement could be added by making successive iterations until life predictions change little and the final choice of $N_{1}$ and $N_{2}$ involves the most damaging events or unless. 
they are recognized as the proper choices according to the engineering judgment of the analyst.

4. Complex service cycle: In Ref: [16] we analyzed a cycle that had originally been discussed in Ref. [23] representing a complex mission cycle for a jet engine. The data for the calculations are shown in Table I. Every block of loading involved 14 events, each of known total strainrange $\Delta c$ and tensile mean stress $\sigma_{0}$, columns (2) and (3). Each event was applied a known number of times, per mission column (4). From these values, iffe was calculated ${ }^{t}$ for each event, column (5). The procedure used then was to choose the cyclic life of the event that produced the lowest life as the $N_{1}$ value, in this case event No. 8 with a life of 2500 cycles. Similarly, the $\mathrm{N}_{2}$ value was the highest one for the events, 64000 cycles for event 6. From these two values, $N_{I}$ and $N_{I I}$ for each event was calculated from the equations shown in Fig. 23. The remaining procedure was identical to that described in earlier paragraphs. The $n / N_{I}$ and $n / N_{I I}$ values for each event were calculated, columns (8). and (9), leading to a calculation of the number of missions required to complete Phase I and Phase II, respectively. Thus, for this problem 79 missions were required to complete Phase I and $200 \mathrm{missions}$ for Phase II, leading to a total of $279 \mathrm{missions}$ to produce fallure.

It is now appropriate to carry the calculations one step further, and revise the procedure to determine $\mathrm{N}_{1}$ and $\mathrm{N}_{2}$ more appropriately. A better choice of $N_{1}$ and $N_{2}$ can be made by considering the higher and

tIn our earlier analysis we used a different formula for life when mean stress is present. Now that we have revised the procedure to include mean stress effects, as discussed in Appendix B, the values in column (5) would be somewhat different. However, since the changes would not seriously affect the numerical values involves, and would have no significance on procedure, we have not remade the calculations for column (5). 
lower of the two life values for the events that produce the largest amounts of damage, rather than the events that produce the highest and. lowest cyclic 11ves.

Using the rationale already discussed, we have added in Table I the column (10) which is not contained in Ref. [23]. After the first calculation is made, as earlier proposed, and the number of missions required to complete each of the two phases are calculated, the damage for each type of event is calculated (column (10)). Events 4 and 8 are the most damaging. Hence we remade the calculations for $N_{1}=2500$ and $N_{2}=5550$. On this basis, events 4 and 8 are still found to be the two most damaging, thus iteration of calculation is not necessary. The total number of missions now calculated is 277. Which differs little from the 279 missions earlier determined, and gives us confidence that we can err considerabiy on the choice of optimum values of $\mathrm{N}_{1}$ and $\mathrm{N}_{2}$ without affecting the final life calculation significantly. However, the degree of error may depend significantly on the type of complex cycle analyzed. For example, if the complex cycling involves a preponderance of damage accumulation at a single life level, errors due to choice of $N_{1}$ and $N_{2}$, or even of choice of damage rule, will become insignificant. Impetus to study cumulative damage rules for complex loading is provided by the extreme high-and low-cycle fatigue operating conditions experienced by components in the NASA Space Shuttie Main Engines (SSME). Several thermal low-cycle fatigue and mechanically induced high-frequency, high-cycle fatigue are superimposed on turbine blades and other critical structural components. One such component, called a Lox Post, is a slender tube carrying cryogenic oxygen through its bore while exposed to high-temperature, hydrogen-rich steam on its exterior. Flow-induced vibration provides the high-cycle fatigue excitation while the thermal transients of engine firing and shut-down contribute to the low-cycle fatigue loadings. To understand better the damage 
interaction in the alloys of construction, cumulative damage experiments were conducted on both 316 SS and a cobalt-base superalloy, Haynes 188 . Results are presented in Refs. [25] to [27]. In view of the improvements (DOCA) to the OCA, we have re-examined both sets of cumulative damage data and have sought optimum material specific constants. Figure 25 shows the results for 316 SS at $1300^{\circ} \mathrm{F}$ compared with calculations by the DDCA, Eq. (6). The exponents $\alpha$ and $B$ from Eq. (6) have been optimized for the data shown, and have values of 0.23 and 0.63 , respectively. Agreement is better than obtained earlier using the original DCA formulation and the single constant, $B=0.4$. The added flexibility of another constant in the DDCA promotes improved accuracy of representation.

Similar treatment, Ref. [27], was given to the analysis of data on Haynes 188 reported by $\mathrm{B} i z$ n, et a1. [26]. The optimum constants are $\alpha=0.35$ and $B=0.60$ in contrast to the universalized values of 0.25 and 0.40 , respectively. Comparison of the DOCA calculations with the experimental results are presented in Fig. 26. A band of expected behavior is shown, reflecting the fact that tests were performed at somewhat different values of $N_{1} / N_{2}$. Again, good agreement is obtained. Most importantly is the unconservative deviation (up to a factor of 10 in cyclic life) of the experimental results from the classical LDR. The nonlinear features of the DDCA (and the OLDR) accurately model the interactions of high-and low-cycle fatigue.

\section{DISCUSSION}

In this re-examination of our procedures for computing damage accumulation we have introduced three major changes.

Restating the DCA by adding a term which enables us to make the damage curve nearly identical with the DLOR that has been found characteristic for a large number of materials in two-level fatigue tests. The new equation is 
called the Double Damage Curve Approach (DDCA). We have also defined more clearly the relation between damage curves and their approximation by the OLDR. Introducing a successive-approximation procedure for the choice of the parameters of Phases I and II of the DLDR.

Modifying the procedure for including mean stress effects in cyclic 11fe. A few comments are appropriate for each of these items. Restatement of Damage Curve Approach and Relation to DLDR

It is important to emphasize that we treat each event on the basis of 11fe level, not the parameters that lead to its life level. The strain range and maximum, minimum, or mean stress are included in the determination of the life level of the event. Thus any appropriate theory can be applied to combined them to determine their life level. Cumulative damage is then calculated in the same manner as it would be at the same life level produced by other parameters leading to this life. This procedure is in contrast to other approaches, e.g. Dubuc, et al. [28] and Bui Quoc [29, 30], which incorporate the complex stress and strain parameters into the expression for the damage function. The advantage of our procedure is that the approach may change for determining the life level without affecting the damage curve analysis. For example, as already discussed, we have progressed to a more accurate and generalized method of treating mean stress, but the change in itself does not alter the DCA.

However, we changed the functional form of the damage curve representation to make it conform more closely to extensive data, and to make it more consistent with the DLDR. The new functional form is called the Double Damage Curve Approach (DDCA). Thus when we make a comparison between results obtained by the DOCA and DLDR analyses we do not have to sort out how much of the difference is due to the simplifications used to establish the 
DLDR, and how much is due to the difference implicit in the two types of damage function used.

The change in our new function is consistent with some of the thinking that has recently been introduced by other investigators. For example, Miller and Zachariah [21] and Miller and Ibrahim [22] devised damage functions which are also based on two stages of damage accumulation - development of shallow surface cracks and propagation of a macroscopic dominant crack leading to failure. In their first effort, damage curves were represented by straight lines on $\log -\log$ coordinates, with breakpoints arranged in similar fashion to ours, as seen in Figs. 23 and 24 ; that is, the line joining the breakpoints had a negative slope. However, in their later work they changed their damage functions so that the slope of the line joining the breakpoints is positive. It is interesting that while the results of damage curve analyses are similar when based on either our curves or those of Miller and his co-workers, the governing parameters are different. If we use crack length as the measure of damage our crack lengths become macroscopic fairly early, while Miller and co-workers derive crack lengths of ultra-microscopic size in the Phase I stage, and macroscopic size only very close to fallure. The interesting point is that, as discussed earlier in the report, the damage accumulation process in going from one damaging curve to another does not depend on all the parameters defining the damage curves, but only on one or two critical constants. Thus they can derive the same final result event though the baseline information differs appreciably from ours.

Successive Approximation Procedures for Choice of $N_{1}$ and $N_{2}$ In our previous publications we recognized the importance of considering the lowest and highest lives to establish the values of $N_{1}$ and $N_{2}$ in the DLDR formulation. A restriction was invoked requiring 1 percent life fraction be imposed before a level could be considered an extreme. We have 
since come to realize that life levels for which the greatest amounts of damage (life fraction, $n / N$ ) are accumulated are also of importance because they represent the major participants in the problem. Thus, we have suggested an iterative process that homes in on the two most damaging life levels and uses them as the $N_{1}$ and $N_{2}$ life levels in the DLDR. Calculations presented in this paper have borne out the accuracy of this approach. Modifying Procedure for Including Mean Stress Effects

The cumulative fatigue damage approaches proposed in this paper do not rely upon how mean stresses affect cyclic life, or the specific mean stress formulation employed. Nonetheless, a cumulative damage analys is cannot proceed until all the life levels can be quantified. Since mean stresses strongly influence fatigue life, a reliable, yet general, mean stress formulation is a necessary adjunct to the cumulative damage approaches. We have modified the mean stress approach from what was used in our earlier publications, and have arrived at a more general means for representing these important effects. It has been demonstrated in Appendix B that a wide range of classical mean stress effects can be accommodated by the newly adopted approach. Because of the approach's flexibility, however, it does require a small amount of fatigue data involving mean stress to evaluate the constants. Evaluation techniques are discussed in Appendix $B$.

\section{CAUTIONS}

While we have been active in developing a procedure for treating damage accumulations either through damage curves or through a DLDR based on these curves, few complex service histories have been treated by these methods. Much experience should be gained in diverse applications before these or other such methods, can be accepted for general use. In many cases, it can be expected that the method will be successful by the very fortuitous nature of such complex loading histories. They can contain many events, but in fact 
most will contribute negligibly to the damage, and life will be governed by one or two of the major loading events. The results of the Teledyne study (in Table I) are typical of what can happen in such cases. Current methods are satisfactory for such cases since they were developed from two load level tests.

The real caution comes in connection with circumstances that have not yet been factored into this or other methods. A partial list of such possibilities is:

\section{Effect of Stress Multiaxiality}

This subject is currently receiving intensive study that is beyond the scope of this paper, and may reveal unusual and difficult-to-predict results, especially when loadings in one set of directions or slip systems are subsequently followed by loadings in other directions favoring other slip and fracture systems.

Deformation Systems in Complex Loading Not Revealed in Individual Loadings Wood and Reimann [31] tested copper in torsion wherein large loading amplitudes for a portion of the life were then followed by lower loading amplitudes. The result did not follow a simpler linear damage rule, Fig. 27(a). Reimann [32] later conducted similar torsion tests of iron. More cycles at the lower strain level were possible after the higher strain level had been applied than if it had not been applied, Fig. 27(b). We have tested copper and steels under similar conditions in axial loadings, but did not reproduce the type of effect observed in torsion. Wood explained his results on the basis that when the larger amplitudes were first applied the strain tended to be absorbed by many slip-planes. Once activated to slip, they remained effective in slipping when the lower amplitude was later applied, even though these planes might not have participated if the lower amplitude loading were applied singly. Thus the plastic strain of the lower loading was 
distributed over more slip planes when preceded by the higher loading, decreasing the strain per slip plane, and increasing the life. Why we have not seen such a phenomenon in the many materials we have tested axially is not clear.

\section{Residual Stresses}

The importance of residual stress has been especially revealed through the recent emphasis on the fracture mechanics of cracked structures. One such experfence is demonstrated by Fig. 28. Two-level load tests were conducted on aluminum in bending [33]. We introduced a notch on only one side of the specimen, and ran the high load first to the point at which a small crack had developed at the root of the notch. The last of the high level loadings applied was with the notch in tension, producing a residual compressive stress at the tip of the crack when the load was removed. When the lower load was applied, the remaining cycle ratio was not less than unity, as normally expected, but it was greater by more than 100 than the initial life of the material at the lower load. The crack had been arrested by the residual compression, and did not progress when the lower load level was applied. The fallure at the low load initiated at the opposite side of the specimen, when there was no notch, and progressed toward the crack that had been started by the high load. The DLDR certainiy was not directly applicable here.

\section{Metallurgical Instabilities}

Stress and strain together with exposure to high temperature reactive gases can produce metallurgical instabilities such as strain aging. Some materials, subjected to strain at high temperature, develop precipitates on the dislocations that alter the subsequent deformation and strength characteristics of the material. Thus loadings which induce such precipitations during one portion of the loading history will reveal a 
behavior in another. portion which by itself would be different because of no metallurgical precipitation. An example is presented below.

While investigation creep-fatigue interaction in the cobalt-base superalloy L-605, at $1400^{\circ} \mathrm{F}$, we observed a strong strain-aging effect [34]. Carbon, initialiy in solution with the matrix, precipitated forming an $\mathrm{M}_{23} \mathrm{C}_{6}$ type carbide along the generated dislocations. As the carbides increased in number and size during cycling, the cyclic stress-strain response of the alloy changed dramatically. The alloy was thus increasing its cyclic flow resistance and altering its resistance to fatigue fallure. The amount of change depended upon several factors - amount and duration of deformation and temperature. The implication to cumulative damage analysis is that the fatigue life at a prescribed load or strain level is not a unique quantity dictated by the magnitude of the loading. Instead, details of prior loadings can alter the fatigue life relation. For example, consider two high temperature loading levels. One under high strains gives rise to dislocation generation and carbide precipitation, producing a life, $N_{1}$. The other is at such a low strain level that no precipitation nuclei are formed, the material does not harden and the ensuing life i.s $N>N_{1}$. If a few high strain cycles are imposed on another sample, carbides will form, the material hardens, and its fatigue resistance will be altered. When the partially fatigued sample is subsequently loaded at the smaller strain level, its fatigue resistance should no longer be associated with $\mathrm{N}_{2}$. Competing processes are occurring simultaneously; fatigue crack nucleation and growth (damage) and material hardening. The difficulty to the analyst is in how to separate these factors to accurately predict remaining fatigue life. Further research remains as to how best to resolve such complex problems.

Another example of altered fatigue resistance at high temperatures is one encountered while studying Strainrange Partitioning for creep-fatigue 
analysis, Ref. [35]. When 316 SS was tested with hold periods at peak compressive stress, an oxide surface layer formed, which cracked during the tensile portion of each cycle. After a few cycles of loading, the surface was riddled with short cracks, most of which hadn't as yet penetrated the substrate. For small cyclic strains, oxides still form, but would not crack during the tensile excursions. Hence, a sample loaded at large strains for a fraction of its life and then fatigued to fallure at a small strain range would experience an additional damaging phenomenon (a cracked oxide surface layer) not accounted for in the original fatigue curve of the alloy at the low strain level. Determining the correct life level for cumulative damage analysis could become a tedious task. Our approach does not specifically address the issue raised above. Instead, the approach is more general and deals only with life levels and not with how the life level is attained.

The phasing of temperature and strain cycling during thermal fatigue can produce additional complications to the problems of cumulative damage assessment. In a study, Ref. [36], of thermomechanical fatigue behavior of the nickel-base superalloy, MAR M 200, we observed significant differences in life depending upon whether in-or out-of-phase cycling was imposed. During in-phase cycling, early developed cracks became filled with oxide while being held open by the tensile stress. This situation is not experienced in the out-of-phase cycling for which compressive stresses close cracks at the high temperature, retarding oxidation within the cracks. Out-of-phase cycling was considerably less damaging than in-phase. Furthermore, the two phasings result in different modes of cracking at the ubiquitous internal carbides. In-phase cycling produced carbide-matrix interfacial cracking while out-of-phase cycling caused the carbides themselves to fracture. The implication of the above findings to cumulative damage analysis are of concern. For example, consider two straining levels, $\Delta \varepsilon_{1}$ and $\Delta \varepsilon_{2}$, 
giving rise to the same cyclic life, $N_{f}$. One condition involves in-phase cycling, $\Delta \varepsilon_{1}$, and the other, out-of-phase, $\Delta \varepsilon_{2}\left[\Delta \varepsilon_{2}>\Delta \varepsilon_{1}\right]$. If cycling of a sample is started at $\Delta \varepsilon_{j}$ and is discontinued after a life fraction $n_{j} / N_{f}$ and is resumed at $\Delta \varepsilon_{2}$ until fallure occurs, the remaining life fraction according to the current rules (and the LDR) would simply be $n_{2} / N_{f}=1-n_{1} / N_{f}$. 1.e., $n_{1}+n_{2}=N_{f}$. Since both conditions produce the same life, any combination would be predicted also to produce the same life. However, it is unlikely that such a simple result would be borne out by experiment, due to different damage accumulation mechanisms existing for the two loading conditions. With different mechanisms of damage, the damage curves of $D$ versus cycle fraction $n / N_{f}$ would not coincide for the two conditions, and hence damage summations would differ from 1.0 .

The lesson to be learned from these examples is that the cumulative damage rules developed in this paper which are based upon a single basic fatigue crack intiation and propagation mechanism, will require refinement for application to unique high temperature conditions wherein additional damage mechanisms can come into play.

\section{CONCLUOING REMARKS}

In this report we have described a philosophy of analysis that has evolved in our laboratories over more than $20 \mathrm{yr}$. Our emphas is has been simplicity of engineering application, and minimizing the baseline information required for implementation. While much of the methodology has been discussed in other reports, this presentation takes the evolution process a few steps further. The format of the damage curve analysis has been altered and the procedure clarified to be consistent with the implied damage curves used in the Double-Linear Damage Rule (DLDR). We call the new formulation the Double Damage Curve Approach (DOCA). We have gained insight as to how to iterate the choice of these substitute damage curves to minimize error while still 
retaining simplicity in analysis. Since our basic damage curves are now consistent in both the DDCA and DLDR analyses, the usefulness of the damage curve analysis procedure has been increased. By making the same calculations through the DDCA we can establish how much error is introduced by the compromises brought about to alter them for OLDR analysis. On the other hand, it also becomes clear from such calculations how much simplicity is gained by the DLOR procedure compared to retention of damage curves with attendant computational complexity.

An important feature of our methods is the characterization of an event only by its life, not the parameters that enter into determining the life. Thus a strainrange and associated mean stress that leads to a given life is treated in identical manner as a smaller strainrange with associated larger tensile mean stress which also leads to the same life. In this way improvements in life calculation per se do not later the equations involved in the DDCA or DLDR analyses. We have, in fact, discussed what we regard to be improvement in accounting for mean stress. Thus, while the life values that enter into a damage calculation may be altered by using the new mean stress relations, the equations operating on these life values do not change.

Experience with the application of the method to a spectrum of complex loading types is currently limited, but we hope to make detalled computations in generic cases to evaluate the effects of various parameters. Sample computations are included in this report.

Finally, we urge caution in the use of the method described, or indeed any other method, to insure that some unexpected phenomenon, not inherentiy contained in the framework of the method, is introduced inadvertently. Among these are stress multiaxiality, unidentified deformation and fracture mechanisms, unknown residual stresses (especially at notches and crack tips), 
oxidation, and metalluigical instabilities.
this report.

Same of

these are

1) lustrated

in 


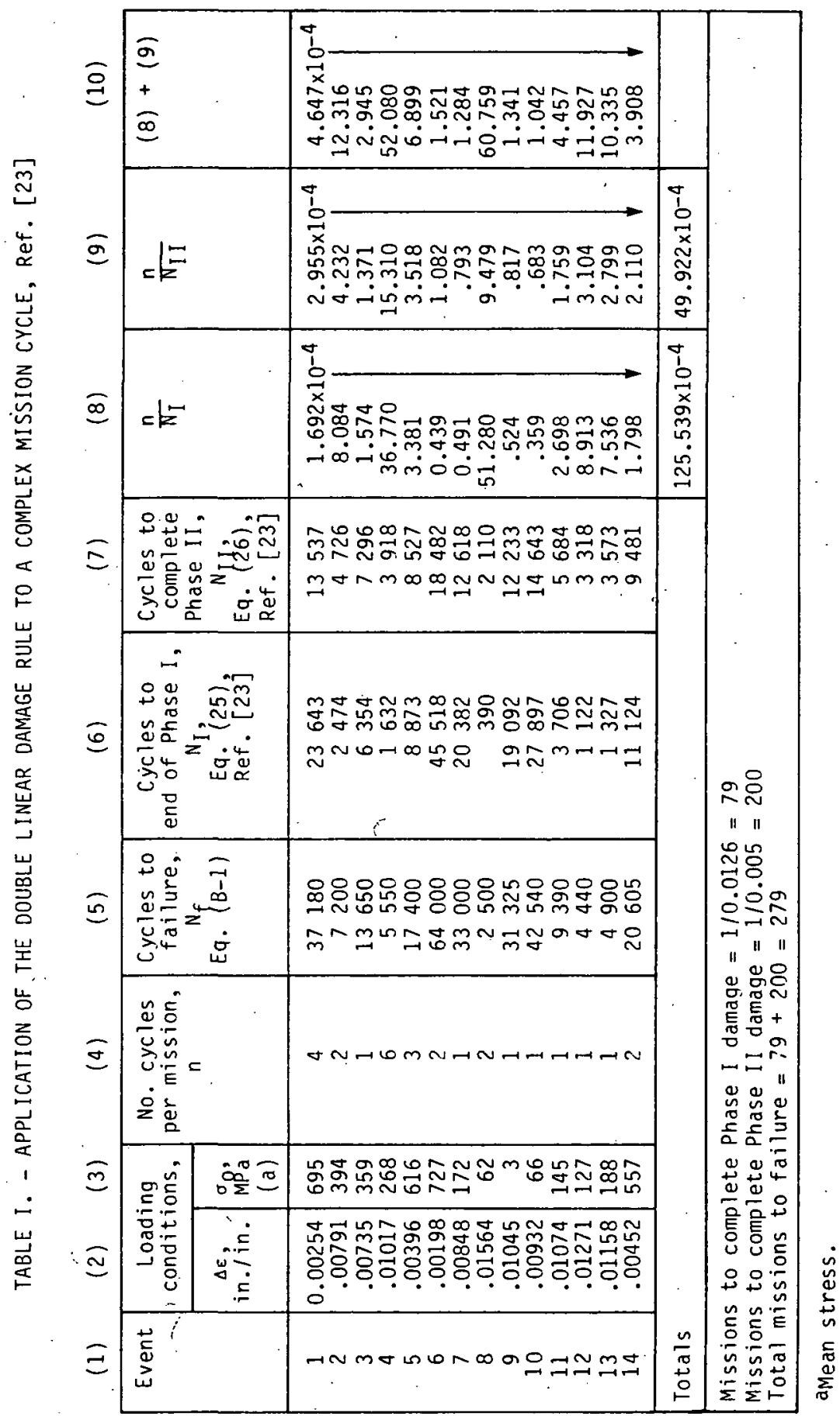


APPENDIX A

DERIVATION OF THE DOUBLE DAMAGE CURVE APPROACH (DDCA)

Our choice of terms to enter the two term damage curve approach was such as to produce a continuous curve coincident with the DLDR at small values of Iife fraction and coincident with the single term OCA equation at large life fractions. The first term is linear in $n / N$ and the second is a power function of $n / N$

$$
D=a_{1}\left[\frac{n}{N}\right]+\left[1-a_{1}\right]\left[\frac{n}{N}\right]^{q_{2}}
$$

where:

$$
\begin{gathered}
a_{1}=\left[0.35\left[\frac{N_{\text {ref }}}{N}\right]^{\alpha}\right] /\left[1-0.65\left[\frac{N_{\text {ref }}}{N}\right]^{\alpha}\right], \alpha=0.25 \\
a_{2}=\left[\frac{N}{N_{\text {ref }}}\right]^{\beta}, B=0.40
\end{gathered}
$$

The coefficient $a_{1}$ is the slope of the first damage accumulation line in the OLDR. The second term in Eq. (A-1) is the same as the original single damage curve term except for a reduction of its coefficient from 1 to $\left(1-q_{1}\right)$ to force conformity with $D=1$ at $n / N=1$. N $N_{\text {ref }}$ is the reference life condition for which damage could be considered to be accumulating linearly toward unity, and $N$ is the life level of interest.

While Eq. (A-1) serves to improve the slope of the damage curve at its origin, especially for large values of $N / N_{\text {ref }}$ (Fig. A-l(a)), additional improvement is required for lower values of $N / N_{\text {ref }}(F i g . A-1(b))$. In both cases, the double term equation deviates the greatest amount in the transition region between the two terms. 
To achieve improved fit over the entire range we resorted to a previously used approach [17] and [18]. Given an equation where the dependent variable is expressed as the sum of two power laws of the independent variable,

$$
y=x^{m}+x^{n}
$$

it is clear that $y \rightarrow x^{m}$ when $x^{n} \ll x^{m}$, and $y \rightarrow x^{n}$ when $x^{m} \ll x^{n}$. Thus $y$ becomes nearly coincident with $x^{m}$ at one extreme of $x$ and nearly coincident with $x^{n}$ at the other extreme. Plotted on log-log coordinates $y$ becomes nearly straight in two regions of $x$. Between the two extreme regions $y$ changes slope gradually from $m$ to $n$. However, we can force $y$ to conform to $x^{m}$ and $x^{n}$ over a wider range, and change more abruptly between the two slopes by rewriting the equation in the form

$$
y^{\gamma}=x^{\gamma m}+x^{\gamma n}
$$

when $x^{m} \gg x^{n}, y$ still approaches $x^{m}$, and similarly when $x^{n} \gg x^{m}, y \rightarrow x^{n}$. The asymptotic lines are thus preserved. However, by the proper choice of $r$ the curve of $y$ versus $x$ can be altered in the range where $x^{m} \cong x^{n}$. Figure A-2 shows the application of this concept for the case at hand. Rewriting Eq. $(A-5)$,

$$
D=\frac{n}{N_{f}}\left[q_{1}^{\gamma}+\left[1-q_{j}^{\gamma}\right]\left[\frac{n}{N_{f}}\right]^{\gamma\left[q_{2}-1\right]}\right]^{1 / \gamma}
$$

If we choose several values of $r$ and plot $D$ versus $n / N$ for $N / N_{\text {ref }}=10$, we get the curves shown in Fig. A-2. It is clear that $r=5$ gives a sufficiently close fit to the double linear damage line, and we have, therefore, tentatively settled on this value. 


\section{APPENDIX B}

\section{MODIFIED PROCEDURE FOR MEAN STRESS EFFECTS}

Service cycles involving varlable amplitude loading will likely encounter mean stresses during portions of the loadings. Since mean stresses significantly alter the expected fatigue life relative to a zero mean stress condition, techniques must be implemented to determine the associated life levels for use with the cumulative damage approaches described in the body of this report. The following describes a promising modified procedure for more reliably dealing with mean stress effects on fatigue life. Consider a material for which the Manson-Coffin-Basquin diagram has already been established, as shown in Fig. B-l(a). No mean stresses are involved in this figure. Figure B-1(b) is exactly the same plot, except that the life scale has changed to reflect the presence of mean stress in accordance with the formulation discussed by

Heidmann [24]. The multiplier on the life scale is $\left[1-\left(\sigma_{0} / \sigma_{f}\right)^{A+B \operatorname{logN}} f\right.$. Although $\sigma_{f} / E$ and $b$ are known from Fig. B-l(a) the constants $A$ and $B$ are at the outset unknown. Several tests involving mean stresses are necessary to determine these constants. As an example, assume these tests are conducted with a mean stress ratio of $0.2,1 . e ., \sigma_{0} / \sigma_{f}=0.2, \sigma_{0}=0.2 \times$ $130=26 \mathrm{ksi}$. For illustration assume that two tests are conducted, one with an alternating stress of amplitude $65 \mathrm{ks} 1$, the other with an alternating stress amplitude of $40 \mathrm{ks}$. The lives are measured, and tabulated as shown in Table B-1. We treat three cases in which the measured lives are assumed to be those in Table B-1. Consider the analysis of the datum for Case I where $\Delta \sigma / 2=130\left(2 N_{f}\right)^{-0.10}=65 \mathrm{ksi}$. The elastic strain amplitude for the alternating stress is $65 / 30 \times 10^{3}=0.002167 \mathrm{in./in}$. We thus proceed to point $P$ in Fig. $B-1(b)$, indicating that the numerical value on the 
horizontal axis is 1024 . Thus we apply this value and the measured life $\left(2 N_{f}=2 \times 55\right)$ to the horizontal scale

$$
1024=(2 \times 55)\left[1-(0.2)^{A+B \log (55)}\right] \frac{1}{(-0.10)}
$$

from which we calculate

$$
A+1.74 B=1.00
$$

If we apply the same procedure to the second datum for Case I, where $\Delta \sigma / 2=40 \mathrm{ksi}$, we develop the equation

$$
A+(3.85) B=1.00
$$

Solving for $A$ and $B$ from Eqs. $(B-2)$ and $(B-3)$.

$$
A=1.0 ; \quad B=0
$$

In a similar manner, if we treat the two measured points for case II, we get

$$
A=2.0 ; \quad B=0
$$

and finally for Case III, the measured life values give

$$
A=3.0 ; B=-0.42
$$

Case I, therefore, represents a material of the Goodman-Morrow type. All the normalized alternating stress/mean stress diagrams consist of a single straight line, as in Fig. B-2. Case II represents a material for which the normalized diagram consists of a single curve of the Gerber type, with convex curvature. Finally, Case III represents a material of general behavior, wherein the normalized alternating stress/mean stress diagram consists of a family of curves, one for each life level. Once the material has been characterized, any corresponding type of plot can be made that suits the needs of the investigator. Figure B-3 shows the mean stress effect for each decade life level between $10^{3}$ to $10^{6}$ cycles.

Figure B-1 can also be used to analyze data wherein the strainrange and mean stress are known. Thus if point $Q$ is known from a total strainrange (which may include appreciable plasticity), we can still establish the 
abscissa at point $R$. If mean stress is also known, together with the values of $A$ and $B$ for an already characterized material, $N_{f}$ can be determined from a simple transcendental equation. Alternatively, if life is known from characterization tests for which $\Delta \varepsilon$ and $\sigma_{0}$ are also known, values of $A$ and $B$ can be computed in the same manner as already 1llustrated. Obviously, for the schematic illustrations, only two tests are needed for determining $A$ and $B$. In practice, many tests may be used to get $A$ and B from a least squares solution. Or, in fact, it may develop that these many tests will define a more general function for $N_{f}$ instead of the relation $A+B \log \left(N_{f}\right)$. 


\section{REFERENCES}

[1] A. Palmgren, Die Lebensdauer von Kugellagern, Verfahrenstechinik (Berlin), 68, 339-341 (1924).

[2] B.F. Langer, Fatigue Failure from Stress Cycles of Varying Amplitude, J. Appl. Mech., 59, A160-A162 (1937).

[3] M.A. Miner, Cumulative Oamage in Fatigue. J. Appl. Mech., 67, Al59-A164 (1945).

[4] N.M. Newmark, A Review of Cumulative Damage in Fatigue. Fatigue and Fracture of Metals, W.M. Murray, ed., Wiley \& Sons, New York, 197-228 (1952):

[5] L. Kaechele, Review and Analysis of Cumulative-Fatigue-Damage Theories: RM-3650-PR, The Rand Corporation, Santa Monica, (1963).

[6] S.S. Manson, Interpretive Report on Cumulative Fatigue Damage in the Low Cycle Range. Welding J. Res. Suppl., 43, 344-S to 352-S (1964).

[7] M.J. O'Neil1, A Review of Some Comulative Damage Theories. ARL/SM-REPORT-326, Aeronautical Research Laboratories, Melbourne, Australia (1970).

[8] 3. Schijve, The Accumulation of Fatigue Damage in Aircraft Materials and Structures. AGARD-AG-157, Advisory Group for Aerospace Research and Development, Paris, France (1972).

[9] J.A. Bennett, A Study of the Damaging Effect of Fatigue Stressing on X4130 Stee1. Proc. ASTM, 46, 693-714 (1946).

[10] G.C. Noll and M.A. Ericson, Allowable Stresses for Steel Members of Finite Life. Proc. Soc. Exp. Stress Anal., 5 [2] 132-143 (1948). See also, Fig. 32 of: S.S. Manson, Avoidance, Control, and Repair of Fatigue Damage. Metal Fatigue Damage-Mechanism, Detection, Avoidance and Repair, ASTM STP-495, S.S. Manson, ed., ASTM, Philadelphia, 254-346 (1971). 
[11] S.S. Manson, A.J. Nachtiga11, and J.C. Freche, A Proposed New Relation for Cumulative Fatigue Damage in Bending. Proc. ASTM, 61, 679-692 (1961).

[12] S.S. Manson, et a]. Further Investigation of a Relation for Cumulative Fatigue Damage in Bending, J. Eng. Ind., 87, 25-35 (1965).

[13] Z. Hashin and A. Rotem. A Cumulative Damage Theory of Fatigue Fa!lure Mater. Sc1. Eng., 34, 147-160 (1978).

[14] S.S. Manson, Interfaces Between Fatigue, Creep, and Fracture. Int. J. Fract., 2, 327-363 (7966).

[15] F.E. Richart, Jr. and N.M. Newmark, An Hypothesis for Determination of Cumulative Damage in Fatigue. Proc. ASTM, 48, 767-800 (1948).

[16] S.S. Manson and G.R. Halford, Practical Implementation of the Double Linear Damage Rule and Damage Curve Approach for Treating Cumulative Fatigue Damage. Int. J. Fract., 17, 169-192 (1981).

[17] S.S. Manson and M.H. Hirschberg, Low Cycle Fatigue of Notched Specimens by Consideration of Crack Initiation and Propagation. NASA TN D-3746 (1967).

[18] S.S. Manson and U. Muralidharan, A Single-Expression Formula for Inverting Strain-Life and Stress-Strain relationships. NASA CR-165347 (1981).

[19] H.J. Grover, An Observation Concerning the Cycle Ratio in Cumulative Damage. Fatique of Aircraft Structures. ASTM STP 274, ASTM, Philadelphia, 120-124 (1960).

[20] S.S. Manson, J.C. Freche, and C.R. Ensign, Application of a Double Linear Damage Rule to Comulative Fatigue. Fatigue Crack Propagation, ASTM, STP-415, ASTM, Philadelph1a, 384-412 (1967). 
[21] K.J. Miller and K.P. Zachariah, Cumulative Damage Laws for Fatigue Crack Initiation and Stage 1 Propagation. J. Strain Anal. Eng. Des., 12, $262-270(1977)$.

[22] K.J. Miller and M.F.E. Ibrahim, Damage Accumulation During Initiation and Short Crack Growth Regimes. Fatigue Eng. Mater. Struct., 4, 263-277 (1981)

[23] 3. Walcher, D. Gray, and S.S. Manson, Structural Design Analysis Aspects of Cold End Rotating Structures. AIAA Paper 79-1190, June 1979.

[24] K.R. Heidmann, Technology for Predicting the Fatigue Life of Gray Cast Iron. Ph.D. Thesis, Case Western Reserve University, Cleveland (1985).

[25] S.S. Manson and G.R. Halford, Complexities of High-Temperature Metal Fatigue: Some Steps Toward Understanding. Isr. J. Technol., 21, 29-53 (1983)

[26] P.T. Bizon, D.J. Thoma, and G.R. Halford, Interaction of High Cycle and Low Cycle Fatigue of Haynes 188 at $1400^{\circ} \mathrm{F}$. Structural Integrity and Durability of Reusable Space Propulsion Systems. NASA CP-2381, 129-138 $(1985)$.

[27] G.R. Halford and S.S. Manson, Reexamination of Cumulative Fatigue Damage Laws. Structural Integrity and Durability of Reusable Space Propulsion Systems. NASA CP-2381, 139-145 (1985).

[28] J. Dubuc, et al., Unified Theory of Cumulative Damage in Metal Fatigue. Weld. Res. Counc. Bu11., No. 162, 1-20 (1971).

[29] T. Bui-Quoc, A Simplified Model for Comulative Fatigue Damage With Interaction Effects. Proceedings of the 1982 Joint Conference on Experimental Mechanics. Society for Experimental Stress Analysis, Brookfield Center, CT, 144-149 (1984).

[30] T. Bui-Quoc, Cumulative Damage with Interaction Effect Due to Fatigue Under Torsion Loading. Exp. Mech., 22, 180-187 (1982). 
[31] W.A. Wood and W. Reimann, Observations on Fatigue Damage Produced by Combinations of Ampittudes in Copper and Brass. J. Inst. Met., 94, 66-70 (i966).

[32] W. Reimann, Some Metallurgical Observations of Cumulative Damage in Fatigue. AFML-TR-68-359 (1968).

[33] L.F. Impellizzeri, Cumulative Damage Analysis in Structural Fatigue. Effects of Environment and Complex Load History on Fatique Life. ASTM STP-462, ASTM, Philadelphia, 40-73 (1970).

[34] S.S. Manson, G.R. Halford, and D.A. Spera, The Role of Creep in High Temperature Low-Cycle Fatigue. Advances in Creep Design. A.I. Smith and A.M. Nicolson, eds., Halsted Press, 229-249 (1971).

[35] S.S. Manson and G.R. Halford, Relation of Cyclic Loading Pattern to Microstructural Fracture in Creep-Fatigue. Fatique 84, C.J. Beevers, ed., Engineering Materials Advisory Services Ltd., Warley, West Midland, England, 1237-1255 (1984).

[36] M.J. Verrilli et al.: A Preliminary Study of the Thermomechanical Fatigue of Polycrystalline MAR M-200. NASA TP-2280 (1984). 
TABLE B-1. - HYPOTHETICAL FATIGUE LIVES UNDER VARIOUS MEANS STRESS CONDITIONS. EXAMPLES USED TO EVALUATE CONSTANTS A AND B IN GENERALIZED MEAN STRESS EQUATION

\begin{tabular}{|c|c|c|c|c|}
\hline & $N_{\text {fo }}$ & & cycle ${ }^{N_{f}} i_{i}$ & \\
\hline \multirow{2}{*}{$\begin{array}{l}\stackrel{\sigma}{a}, \\
k s i\end{array}$} & \multirow{2}{*}{$\sigma_{0}=0$} & \multicolumn{3}{|c|}{$\sigma_{0}=26 \mathrm{ksi}$} \\
\hline & & Case I & Case II & Case III \\
\hline $\begin{array}{l}+65 \\
\mp 40\end{array}$ & $\begin{array}{r}512 \\
65 \quad 736\end{array}$ & $\begin{array}{r}55 \\
7058\end{array}$ & $\begin{array}{r}340 \\
43704\end{array}$ & $\begin{array}{r}327 \\
15 \quad 332\end{array}$ \\
\hline \multicolumn{5}{|c|}{ Computed } \\
\hline $\begin{array}{l}A \\
B\end{array}$ & - & $\begin{array}{c}1.00 \\
0\end{array}$ & $\begin{array}{l}2.00 \\
0\end{array}$ & $\begin{array}{r}3.00 \\
-.42\end{array}$ \\
\hline
\end{tabular}




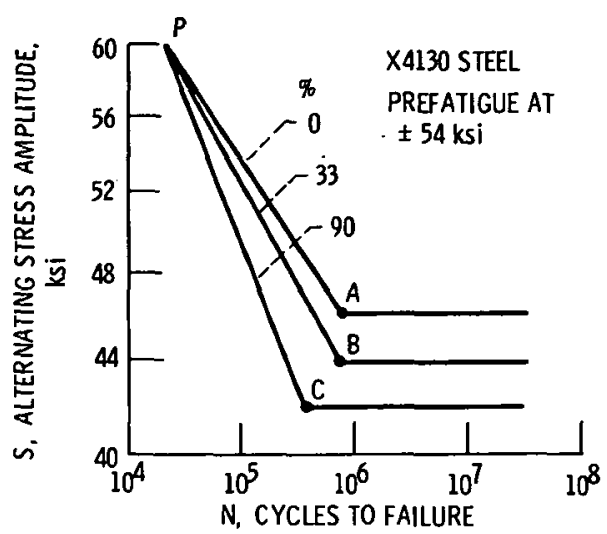

Fig. 1. Fatigue curves for specimens prefatigued at $\pm 54 \mathrm{ksi}$ for 0,33 , and $90 \%$ of crack initiation life. After Bennett (9).

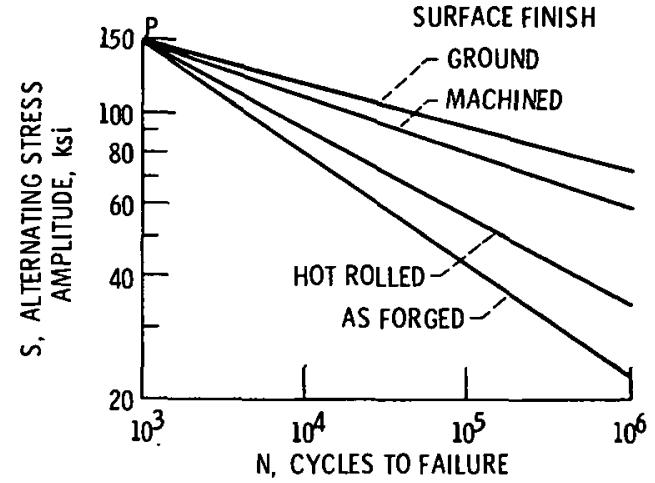

Fig. 2. Surface finish effect on fatigue of medium strength steel. After Noll and Erickson (10).

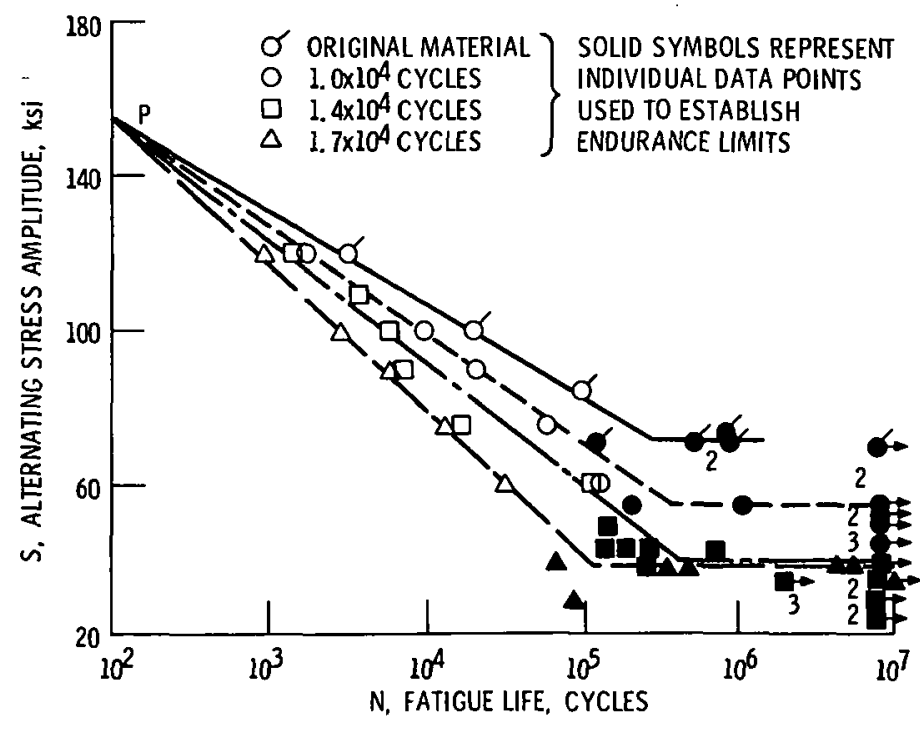

Fig. 3. Comparison of $S-\log N$ lines for original and pre-stressed SAE 4130 heat treated material. Cyclic pre-stress conditions were $100 \mathrm{ksi}$. Median points shown in all cases. After Manson, Nachtigall and Freche (1l). 


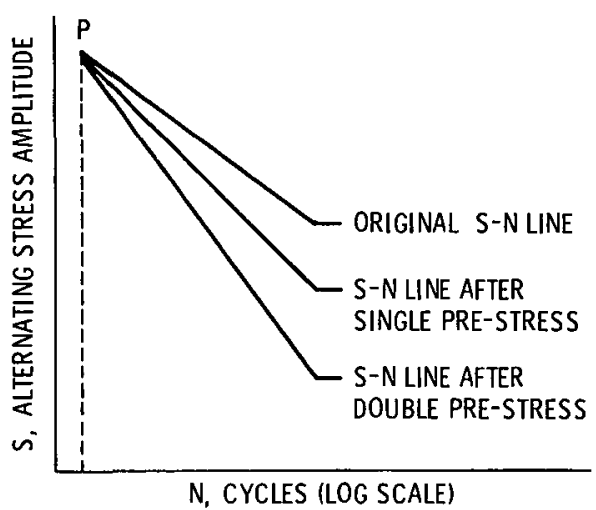

Fig. 4. General location of S- $\log N$ lines for original material and materials subjected to a single and double pre-stress condition after Manson, Nachtigall, and Freche (11).

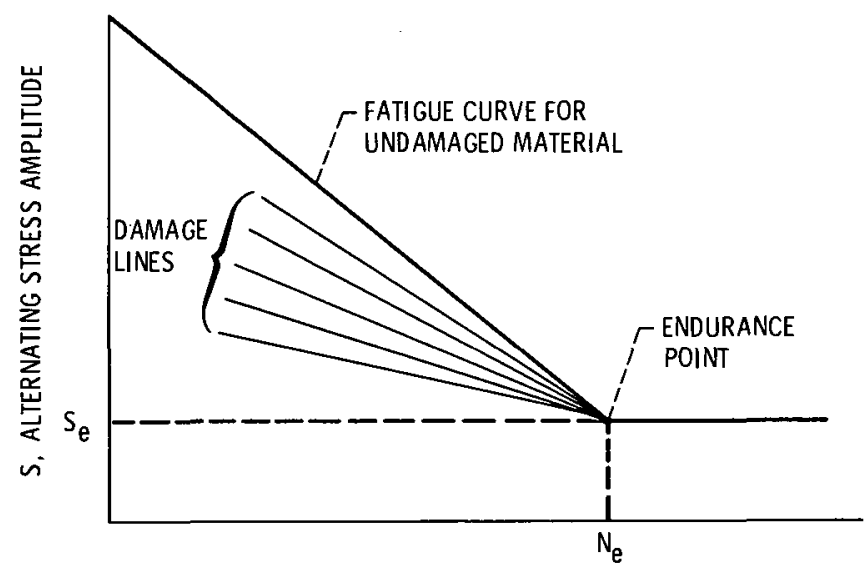

$\mathrm{N}$, CYCLES TO FAILURE (LOG SCALE)

Fig. 5. Damage lines converging at the endurance limit. After Hashin and Rotem (13).

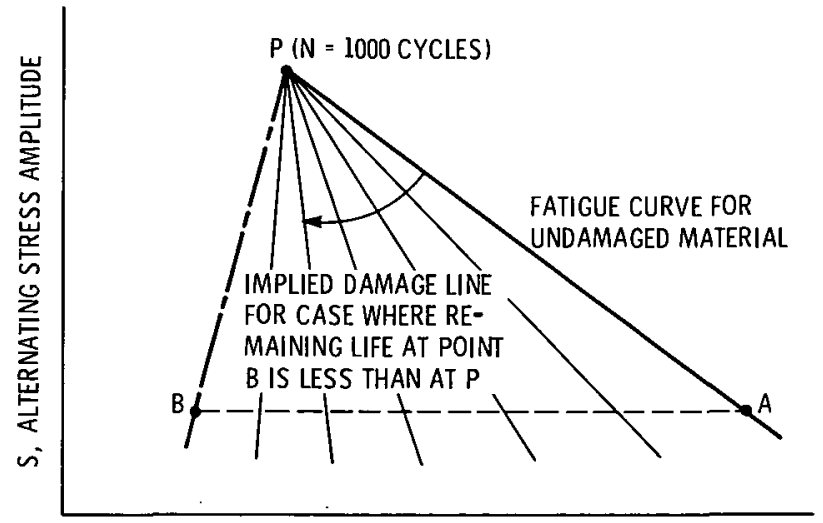

N, CYCLES TO FAILURE

Fig. 6. Example of physically unacceptable damage line obtained by rotation about convergent point only. 


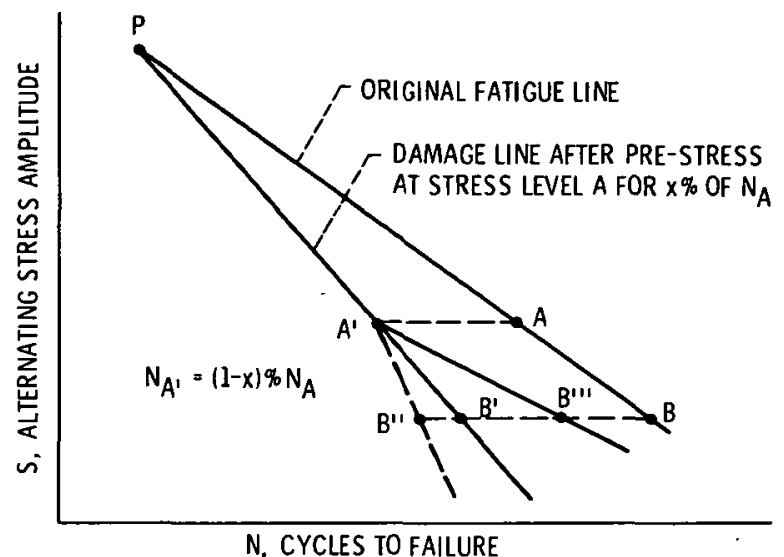

Fig. 7. Convergence approach over-predicts remaining life at stress level $B$.

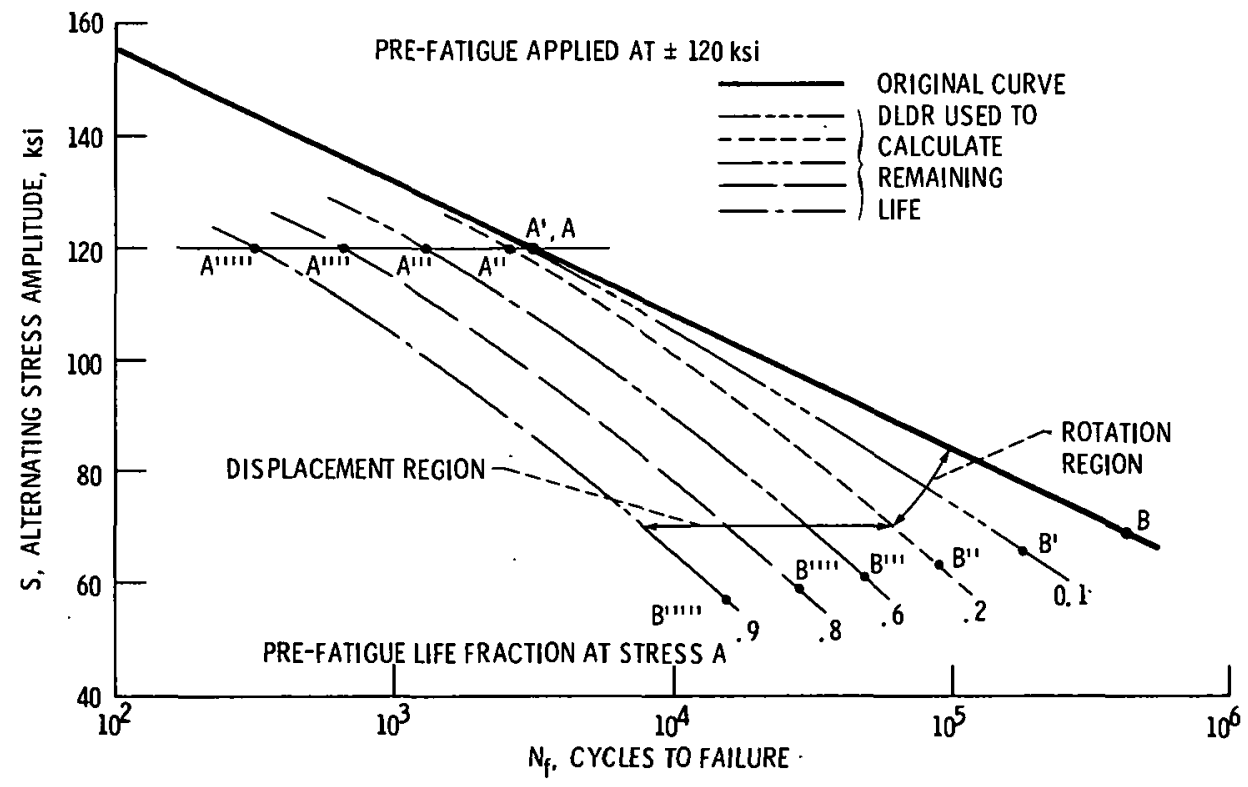

Fig. 8. Rotation and displacement of fatigue damage lines calculated using the double linear damage rule. 


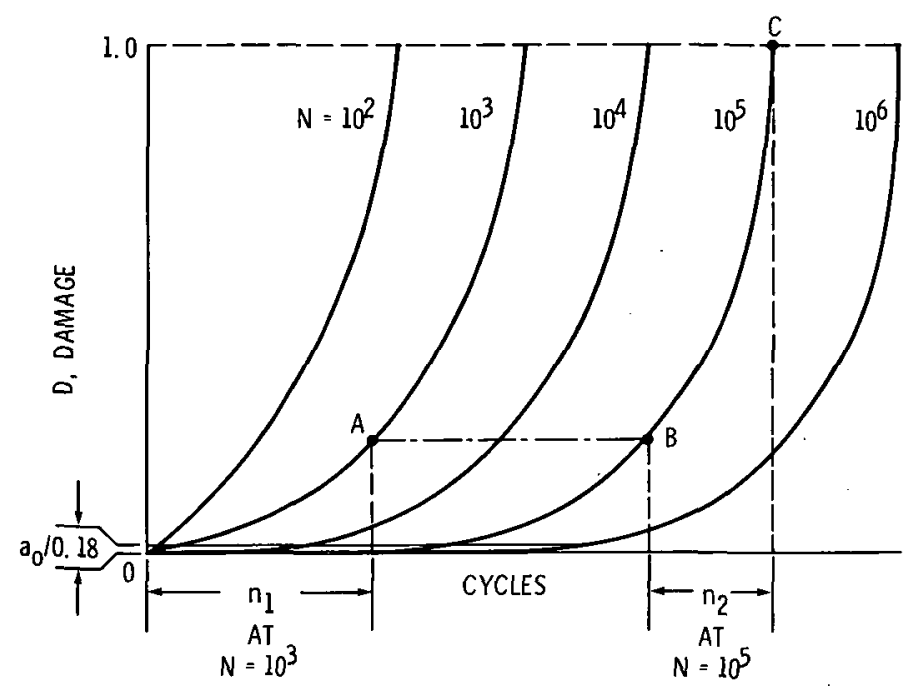

Fig. 9. Schematic of damage accumulation curves based on early crack growth equation.

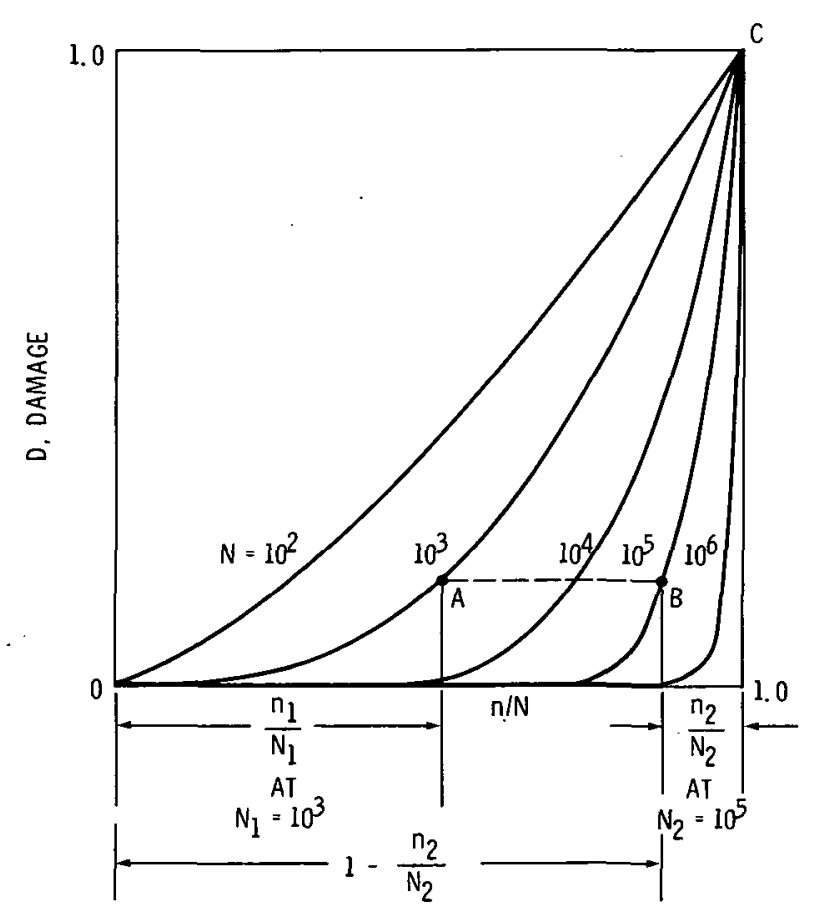

Fig. 10. Schematic of normalized damage accumulation curves. 

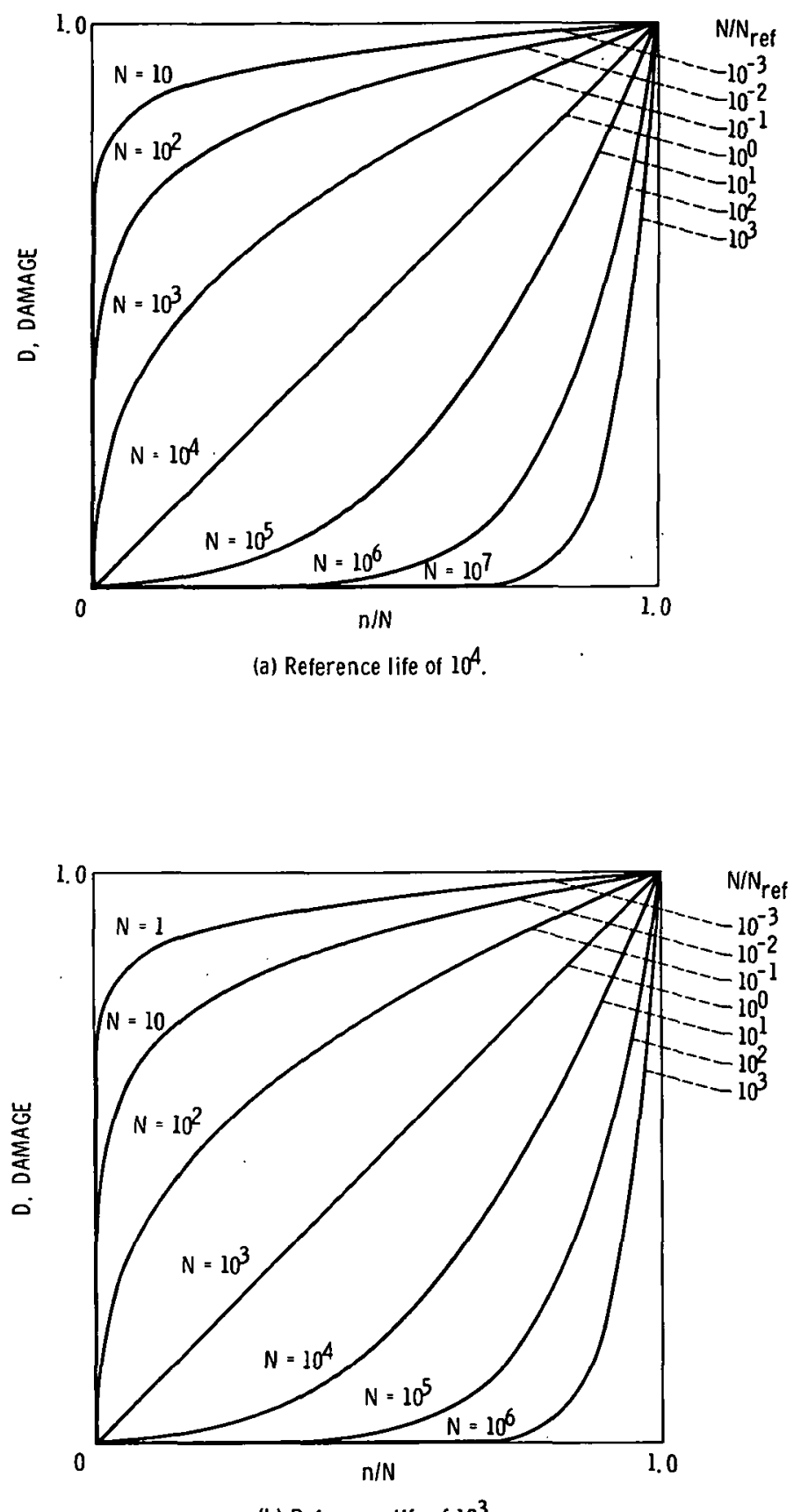

(b) Reference life of $10^{3}$.

Fig. 11. Damage curves. 


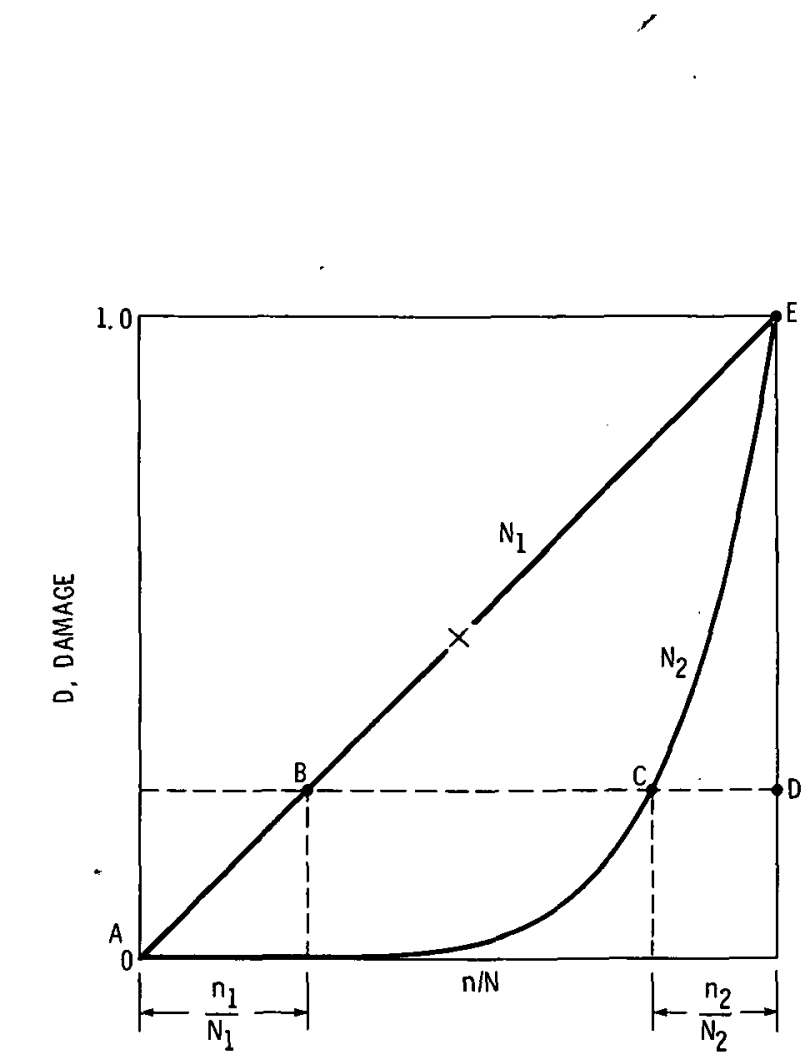

(a) Two life levels, $N_{1}<N_{2}$.

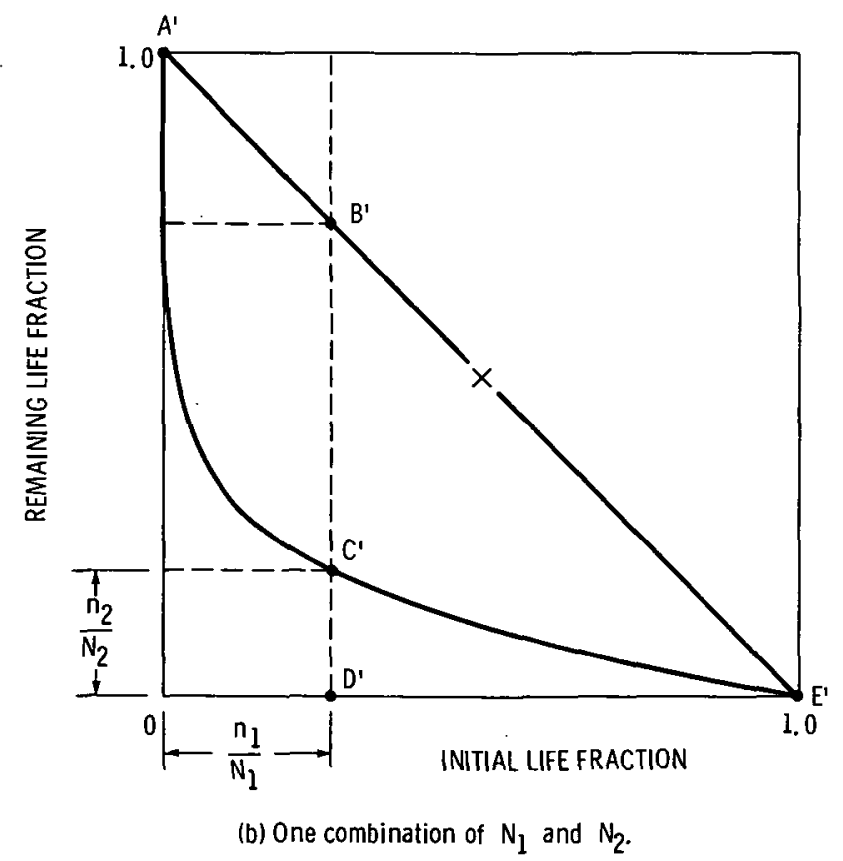

Fig. 12. Damage curves and damage interaction. 


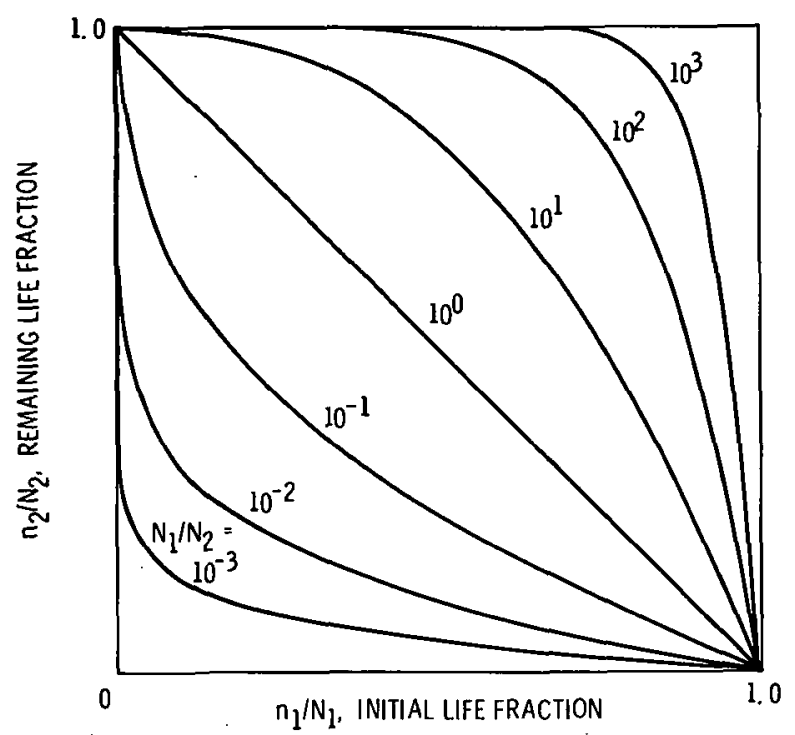

(c) All combinations of $\mathrm{N}_{1}$ and $\mathrm{N}_{2}$.

Fig. 12. Concluded.

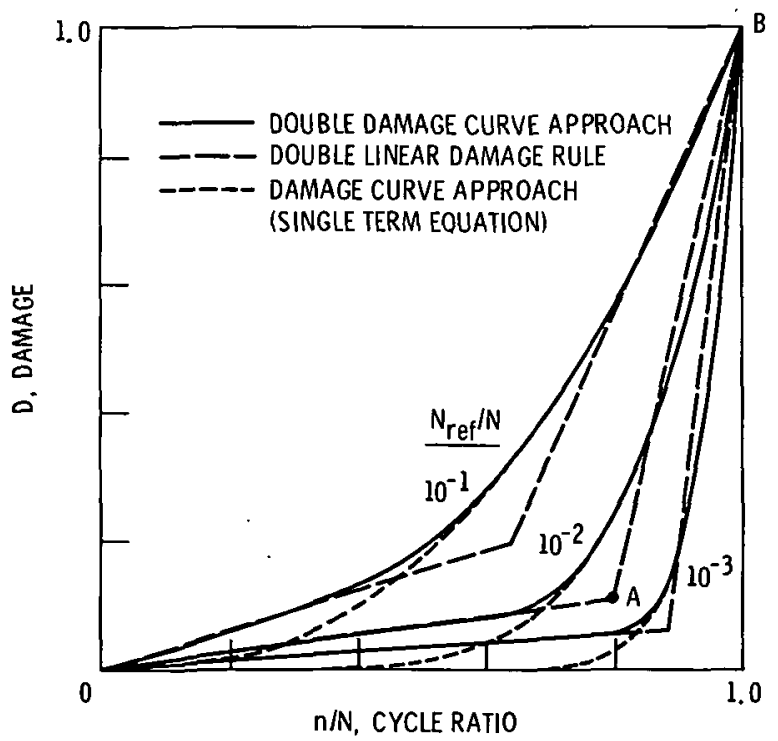

Fig. 13. Double damage curve approach (DDCA) blends the DLDR at low cycle ratios with the DCA at high cycle ratios. 

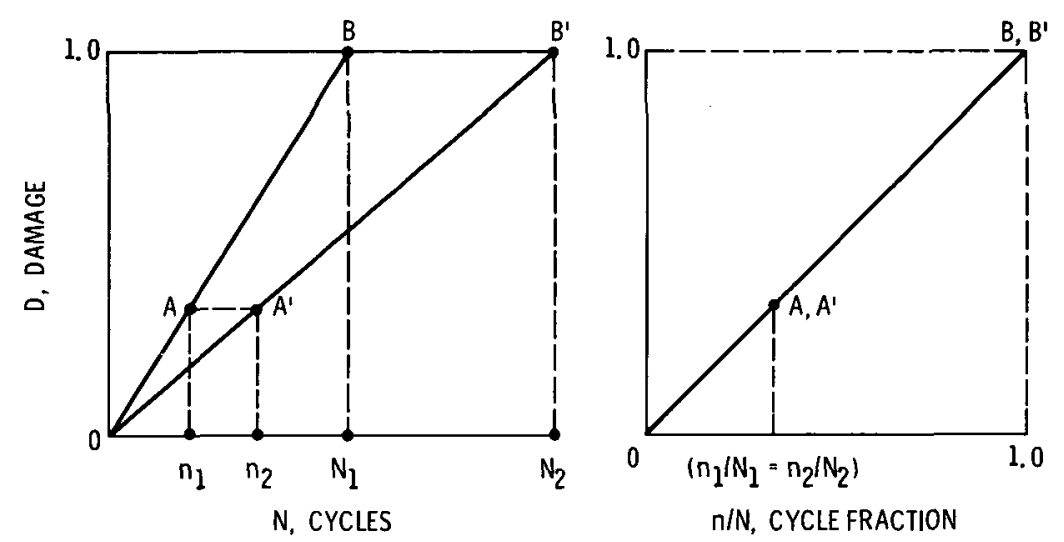

(a) Linear damage.
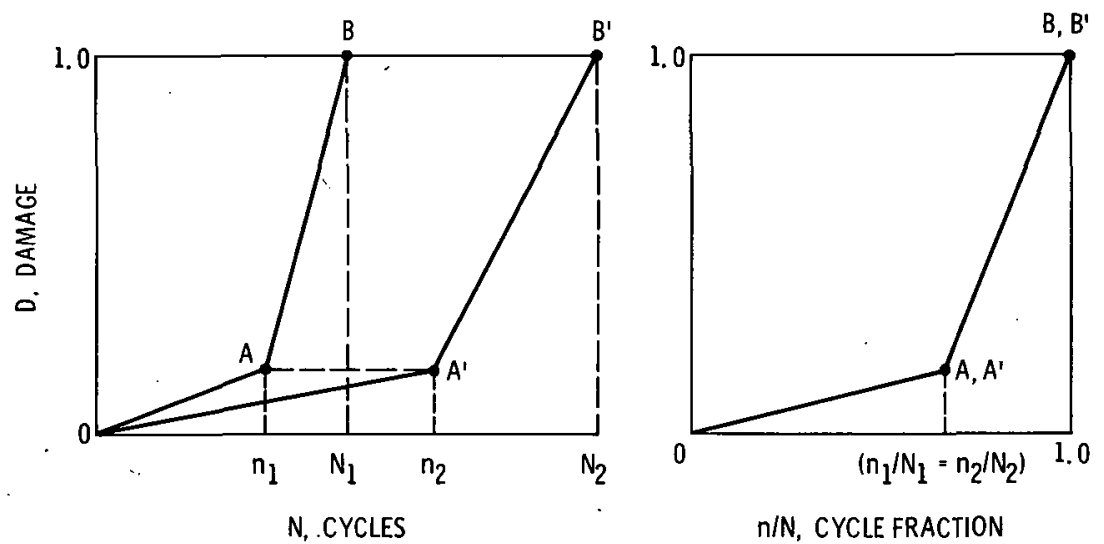

(b) Double-linear damage.
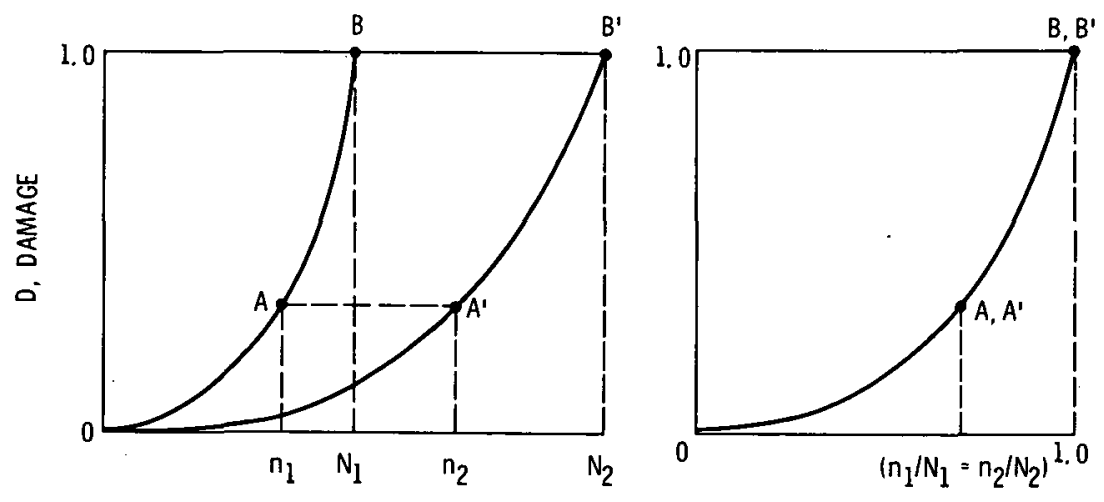

N, CYCLES

N/N, CYCLE FRACTION

(c) General non-linear damage.

Fig. 14. Damage accumulation leading to linear damage rule. 


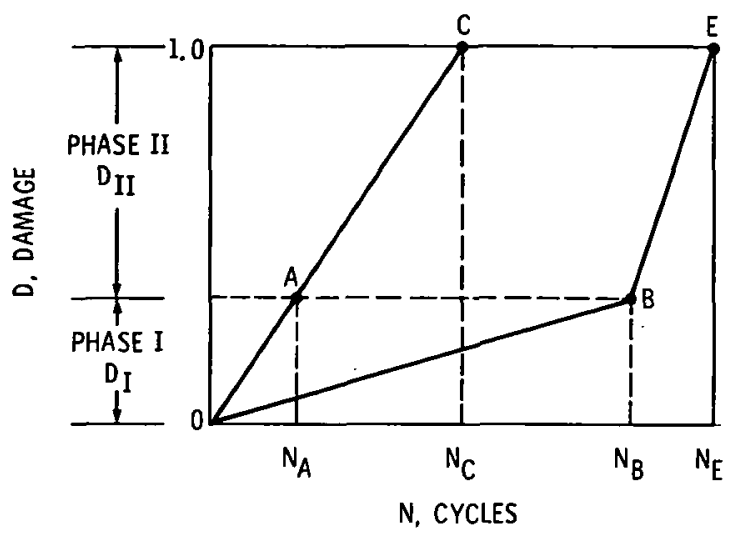

(a) Damage accumulation curves.

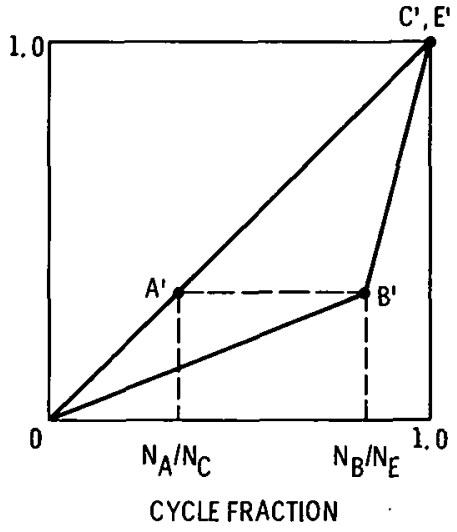

(b) Double-linear damage rule leading to non-linear life fraction summations.

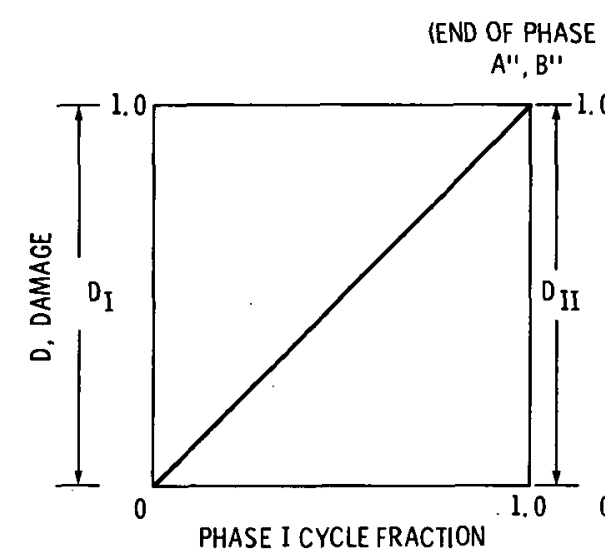

(END OF PHASE II) C". E"

(c) Linear life fraction rule for
phase I damage accumulation.

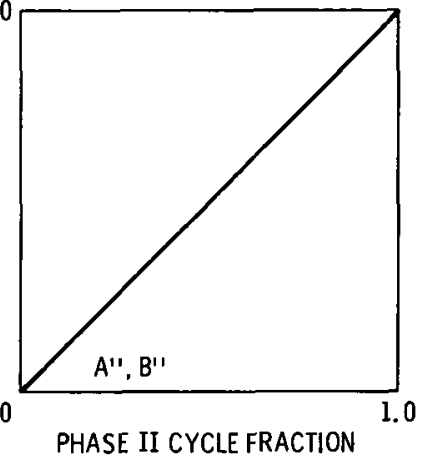

(d) Linear life fraction rule for phase II damage accumula tion.

Fig. 15. Elements of double-linear damage accumulation.
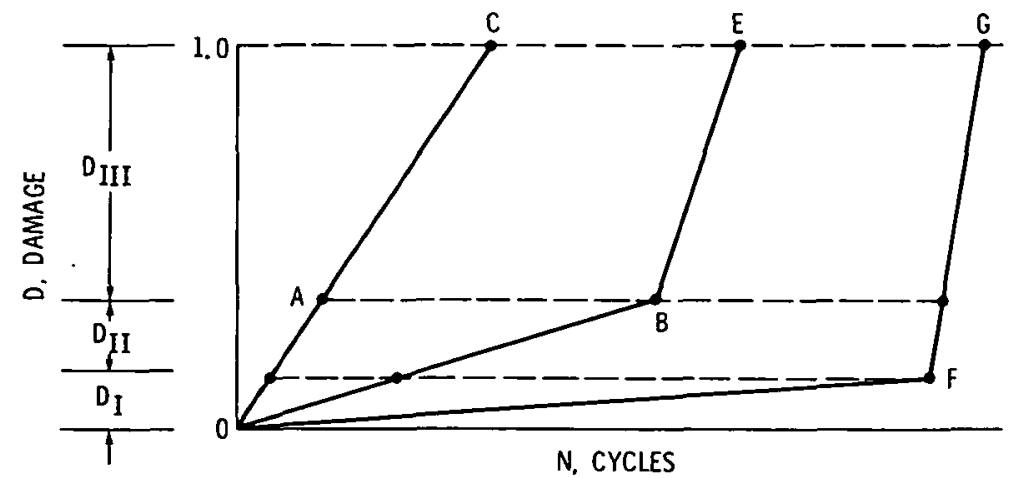

(a) Damage accumulation curves.

Fig. 16. Elements of triple-linear damage accumulation. 


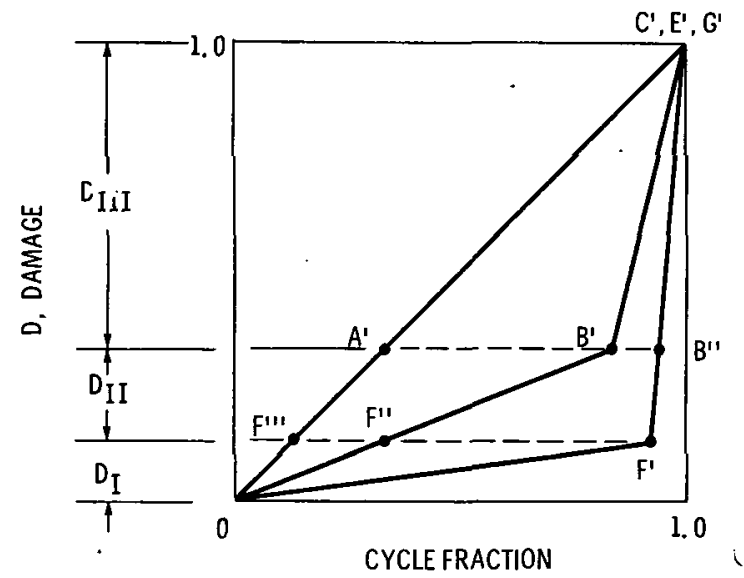

(b) Triple-linear damage curves leading to non-linear life fraction summations.

Fig. 16. Continued.
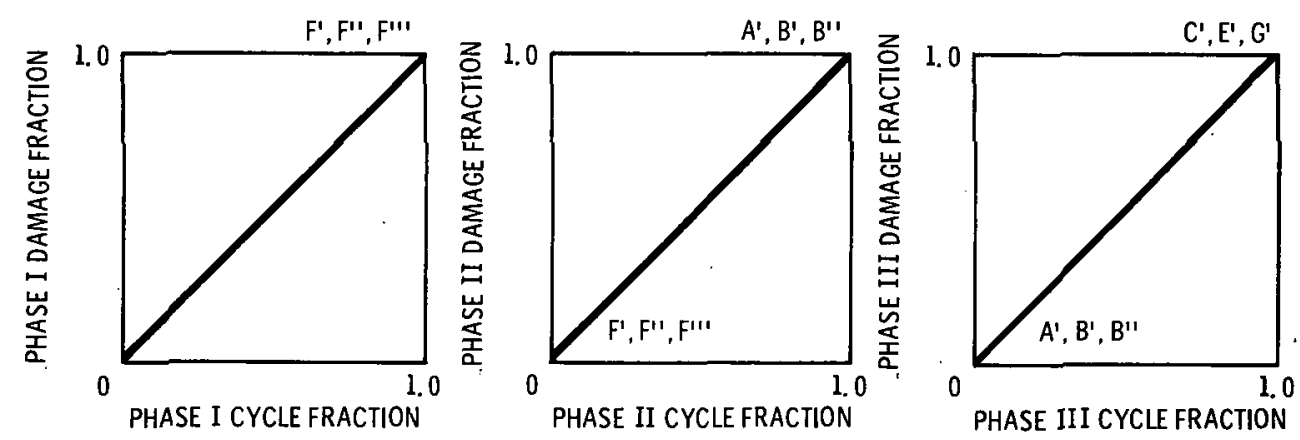

(c) Triple-linear damage rule.

Fig. 16. Concluded.

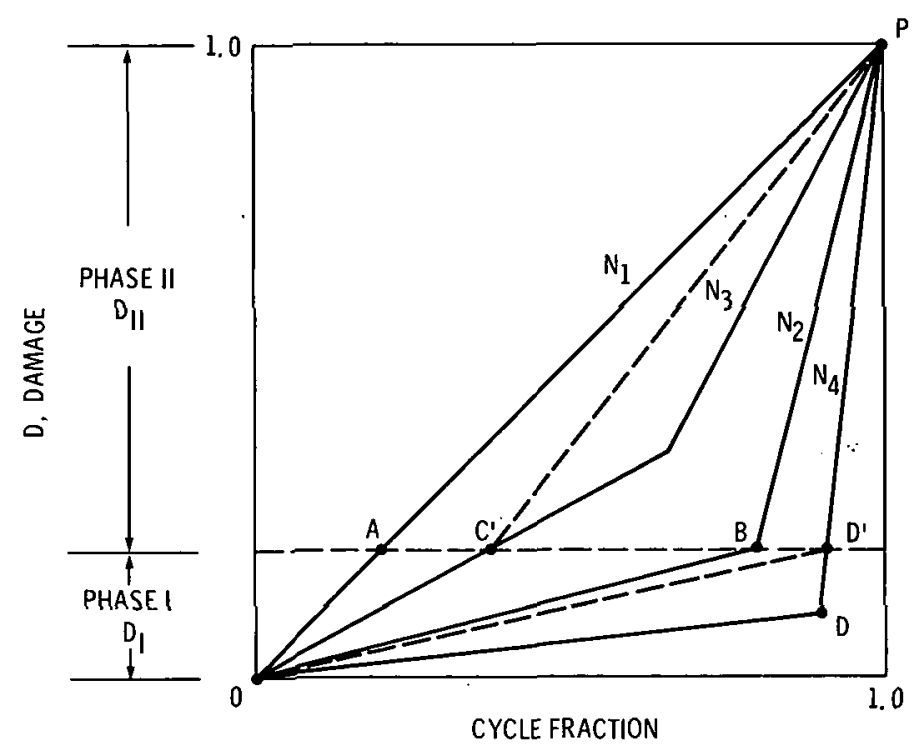

Fig. 17. Conservative approximation leading to a double-linear damage rule when breakpoints in damage curves are not at the same level. 


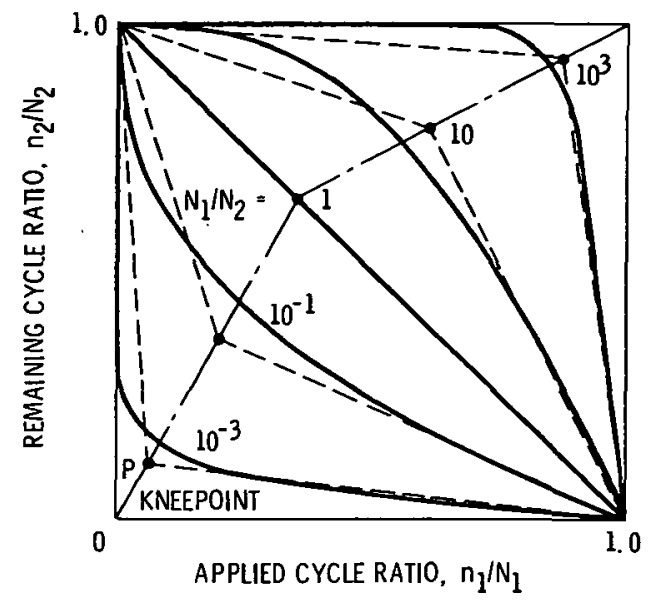

Fig. 18. Cycle ratio relationship for two level tests as deduced from damage curve approach, and replacement of damage curves by two straight line segments to create a double linear damage rule.

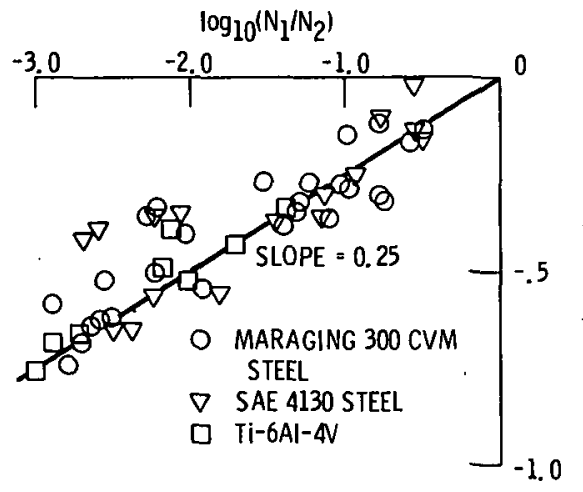

(a) Exponent.

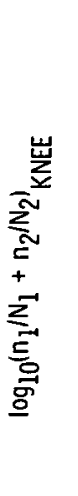

崖

ig. 19. Determination of exp double linear damage rule.

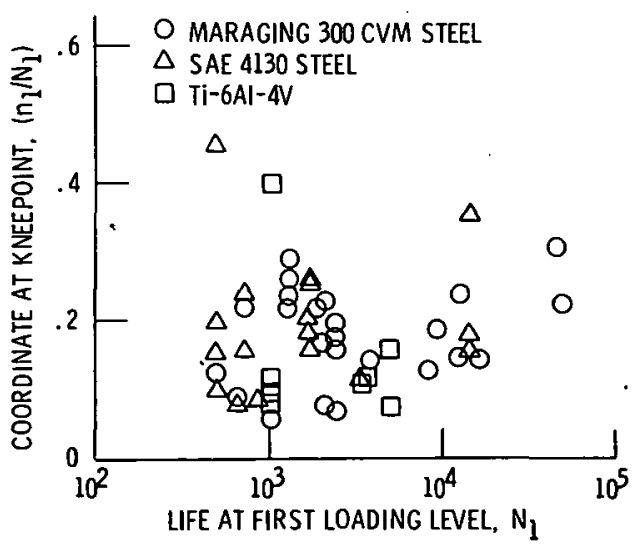

Fig. 20. Lack of correlation between knee point coordinates and the concept of a discrete crack initiation event. 


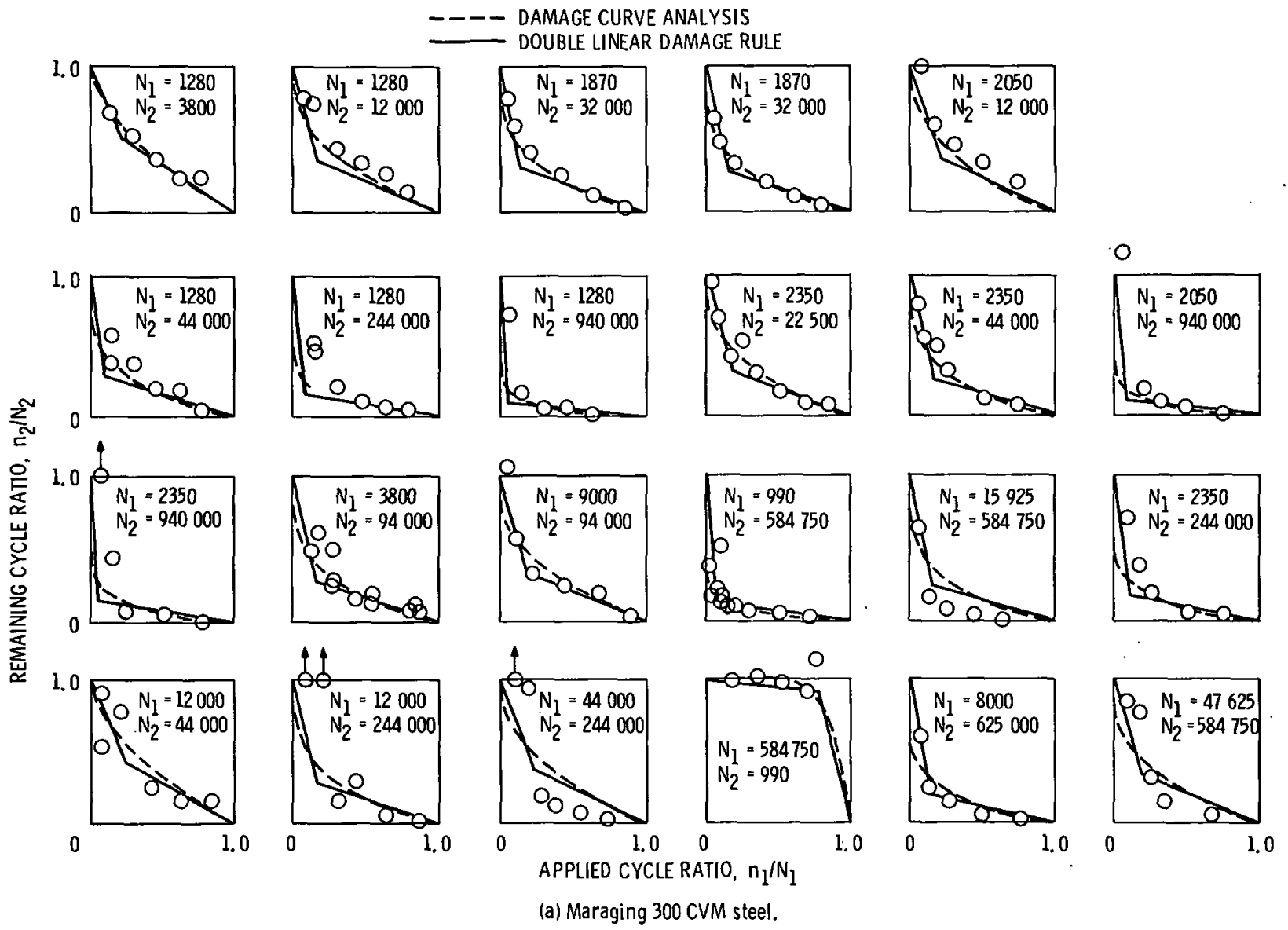

Fig. 21. Two load level test results for three engineering alloys showing comparison with predictions by the damage curve approach and the double linear damage rule. Data from Manson, Freche, and Ensign (20) and previously unpublished NASA results. 

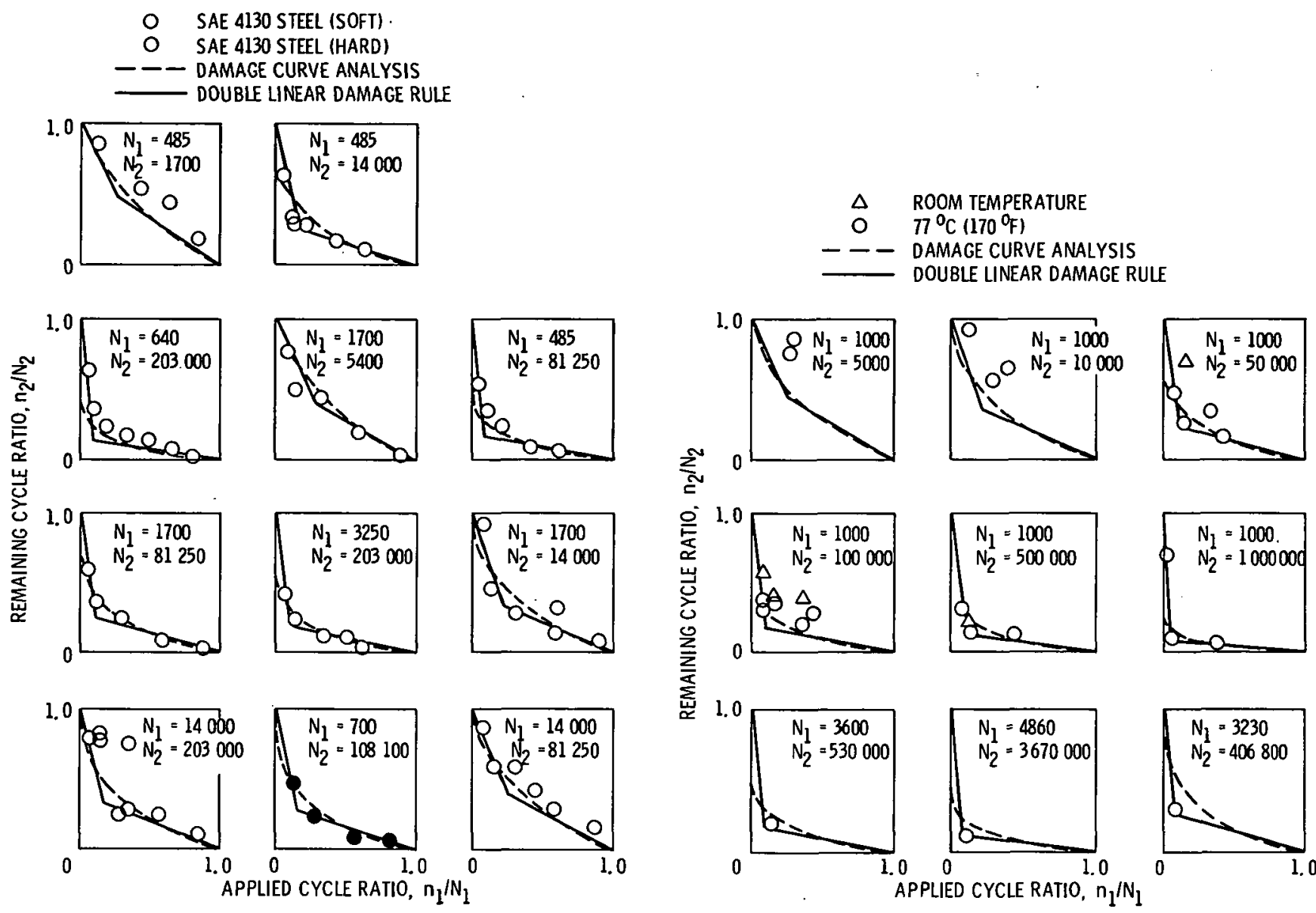

(b) SAE 4130 steel.

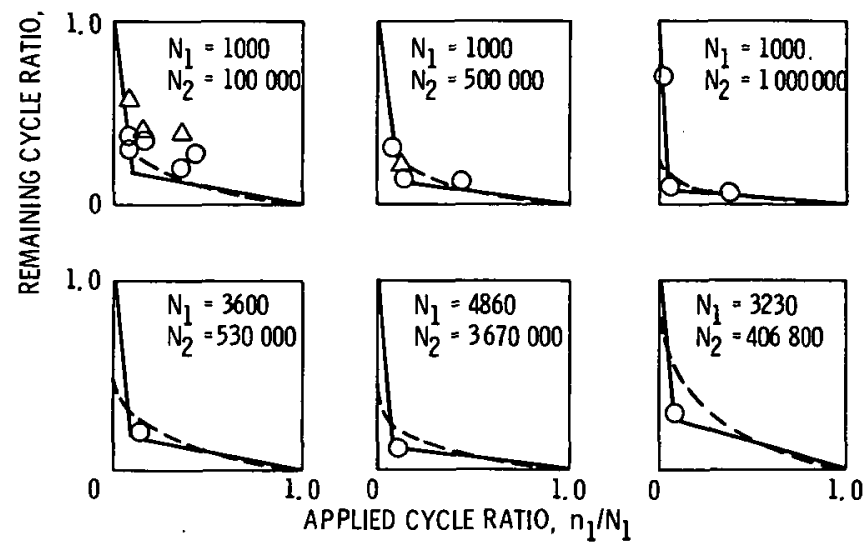

(c) Titanium alloy $6 \mathrm{Al}-4 \mathrm{~V}$.

Fig. 21. Conciuded.

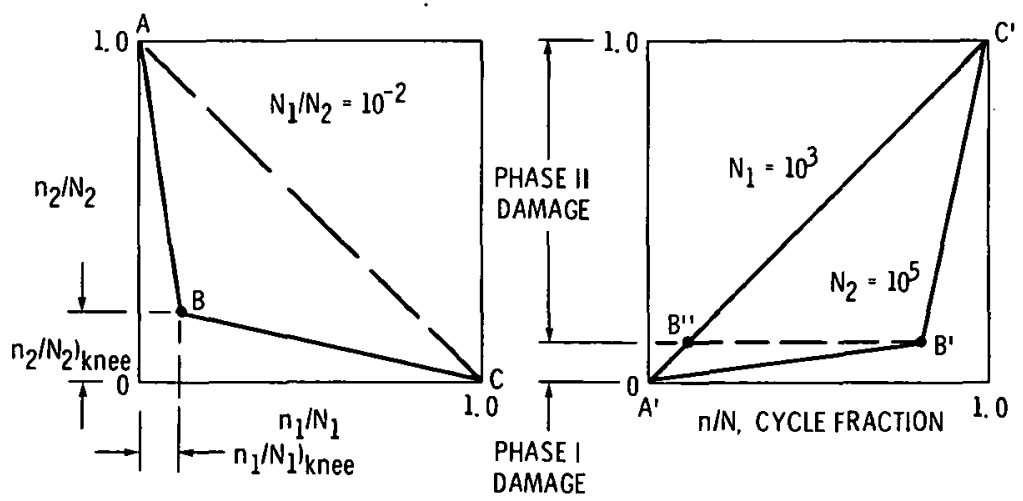

(a) Conventional interaction lines.

(b) Reinterpretation as damage lines.

$$
\begin{aligned}
& N_{1}=1000 ; N_{I, 1}=111 ; N_{I I, 1}=889 \\
& N_{2}=100,000 ; N_{I, 2}=79,445 ; N_{I I, 2}=20,555 \\
& \text { Applying } 16 \text { of single loading life in blocks to failure: } \\
& B_{I}(10 / 111)+(1000 / 79,445)=1.0 ; B_{I}=9.74 \text { alocks } \\
& { }^{B_{I I}}(10 / 889)=(1000 / 20,555)=1.0 ; B_{I I}=16.67 \text { Blocks } \\
& \text { Based on DLDR, Total Blocks at Failure }=B_{I}+B_{I I}=26.41 \\
& \text { Based on Miner Linear Damage Rule, rotal Blocks at Failure }=\text { so. }
\end{aligned}
$$

Fig. 22. Double linear damage rule applied to block loading involving two loading levels. 


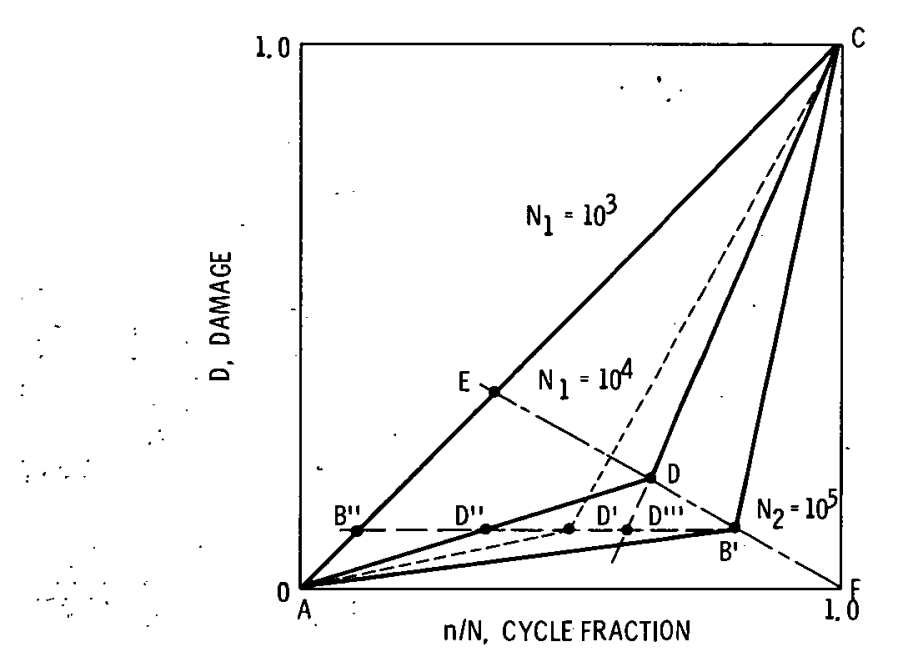

Applying $1 \%$ of single loading life in blocks to failure:

$\therefore \quad N_{1}=N \exp (2 N)$
$4=\frac{1}{\ln \left|\frac{N_{1}}{N_{2}}\right|} \ln \left\{\frac{\ln \left[0.35\left(N_{1} / N_{2}\right)^{0.25}\right]}{\ln \left[1-0.65\left(N_{1} / N_{2}\right)^{0.25}\right]}\right\}$

$Z=\frac{\ln \left[0.35\left(\mathrm{~N}_{1} / \mathrm{N}_{2}\right)^{0.25}\right]}{\mathrm{N}_{1}^{*}}$.

For $\mathrm{N}_{1}=10^{3}, \mathrm{~N}_{2}=10^{5}$, and $\mathrm{N}_{3}=10^{4}$ :

$\mathrm{N}_{1,3}=4,904$

$\mathrm{N}_{11,3}=5,092$

From Fig. 20, $\mathrm{N}_{1 y}=111$, and $\mathrm{N}_{1 \zeta 1}=889$

$\mathrm{N}_{12}=79,445$ and $\mathrm{N}_{112}=20,555$

B $(10 / 111)+(100 / 4,900)+(1000 / 79,445) .=1.0 ; \quad B_{I}=8.13$ Blocks

${ }_{\text {BI }}(10 / 889)+(100 / 5,092)+(1000 / 20,555)=1.0 ; \quad B_{I I}=12.57$ Blocks

Based on DLDR, Total Blocks at Failure $=B_{I}+B_{1 I}=20.7$

Based on Miner Linear Damage Rule, Total Blocks at Faflure $=33.3$

Fig. 23. Double linear damage rule applied to block loading involving three loading levels. Third level intermediate to nominal extreme life levels $\mathrm{N}_{1}$ and $\mathrm{N}_{2}$.

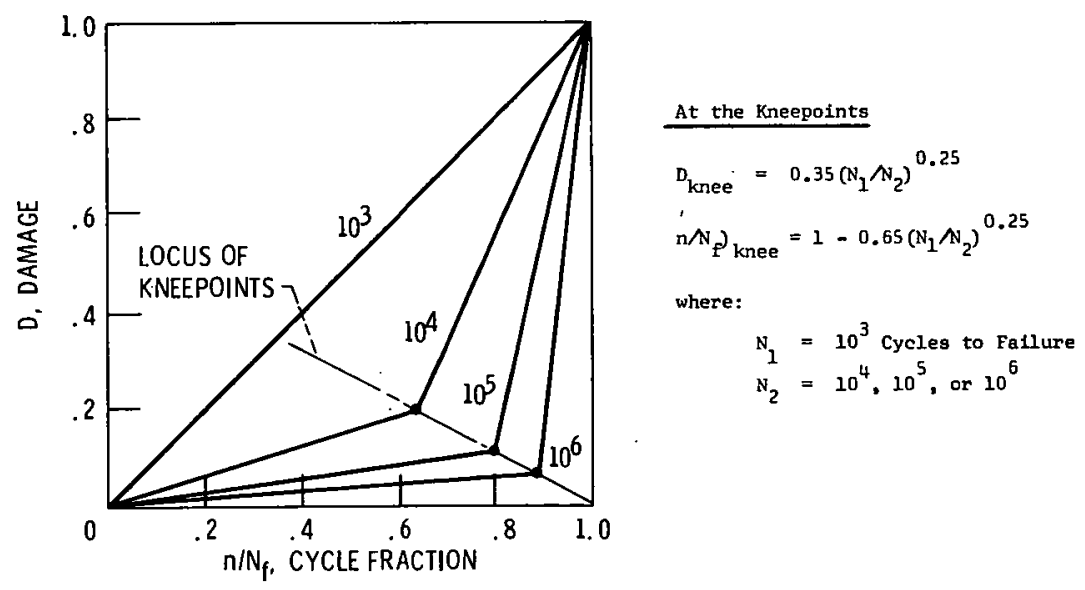

(a) Representation of double linear damage rule by damage lines.

Fig. 24. Double linear damage rule applied to block loading involving four loading levels, 
ORIGIMAL PAQE

OF. POOR QUALITY?
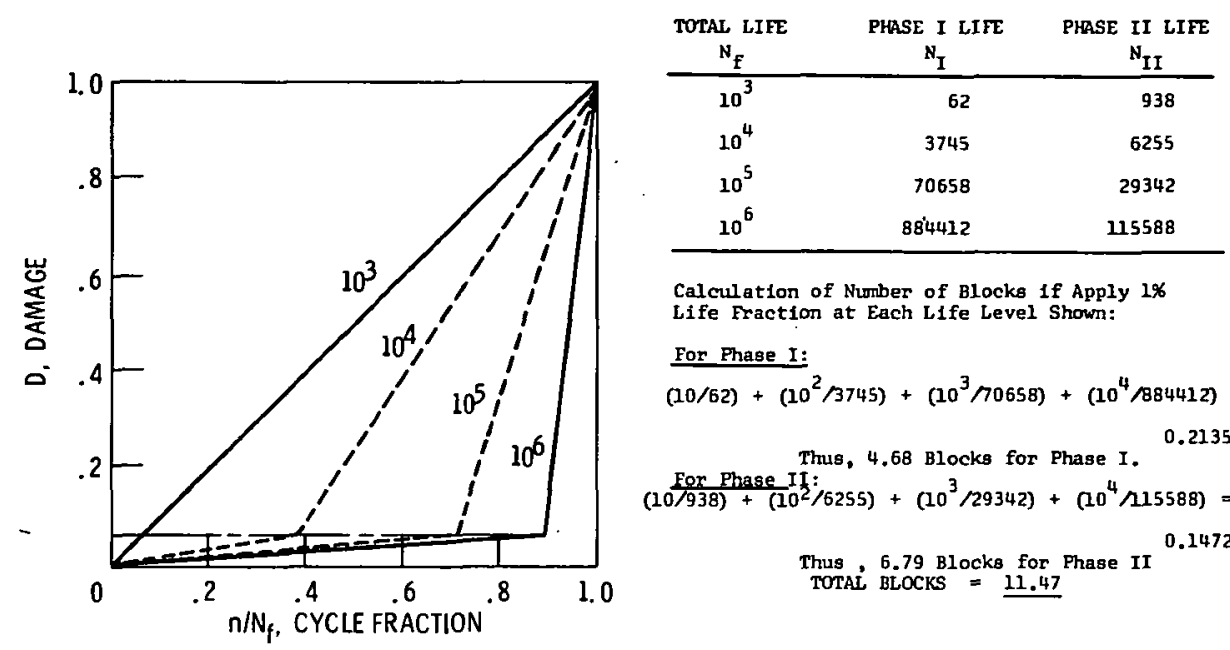

Calculation of Number of Blocks if Apply $1 \%$ Life Fraction at Each Life Level Shown:

For Phase $\mathrm{I}$ :

$(10 / 62)+\left(10^{2} / 3745\right)+\left(10^{3} / 70658\right)+\left(10^{4} / 884412\right)=$ Thus, 4.68 Blocks for Phase $I$. 0.2135 Fox Phase II: Thus, 6.79 Blocks for Phase II 0.1472 Thus 6.79 Blocks for
TOTAL BLOCKS $=\underline{11.47}$

(b) Application of double linear damage rule with $\mathrm{N}_{1}=10^{3}$ and $\mathrm{N}_{2}=10^{6}$.

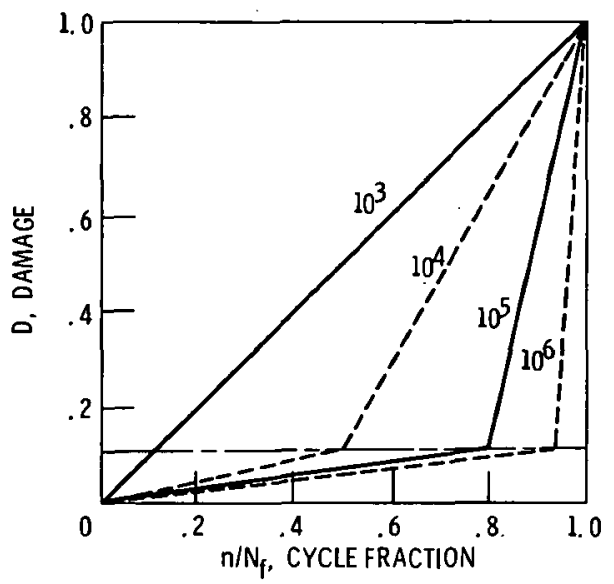

\begin{tabular}{ccc}
$\begin{array}{c}\text { TOTAL LIFE } \\
N_{f}\end{array}$ & PHASE I LIFE & PHASE II LIFE \\
\hline $10^{3}$ & 111 & N $_{\text {II }}$ \\
$10^{4}$ & 4910 & 889 \\
$10^{5}$ & 79445 & 5090 \\
$10^{6}$ & 928300 & 20555 \\
\hline Calculation of Number of Blocks if Apply $1 \%$
\end{tabular}
Life Fraction at Each Life Level Shown:

For Phase I: $(10 / 111)+\left(10^{2} / 4910\right)+\left(10^{3} / 79445\right)+\left(10^{4} / 928300\right)=$

Thus, 7.47 Blocks for Phase I 0.1338 $\frac{\text { For Phase II: }}{(10 / 889)+\left(10^{2} / 5090\right)+\left(10^{3} / 20555\right)+\left(10^{4} / 71700\right)}=$ Thus, 4.56 Blocks for Phase II 0.2191 TOTÁL BLOCKS $=\underline{12.03}$

(c) Application of double linear damage rule with $\mathrm{N}_{1}=10^{3}$ and $\mathrm{N}_{2}=10^{5}$.
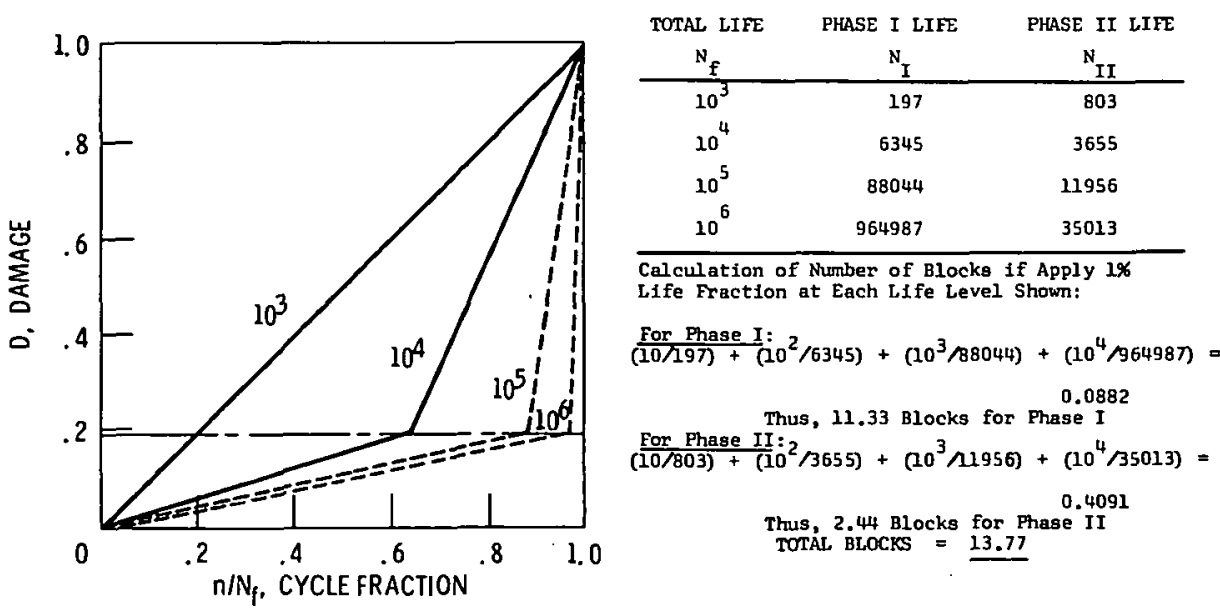

(d) Application of double linear damage rule with $N_{1}=10^{3}$ and $N_{2}=10^{4}$.

Fig. 24. Concluded. 


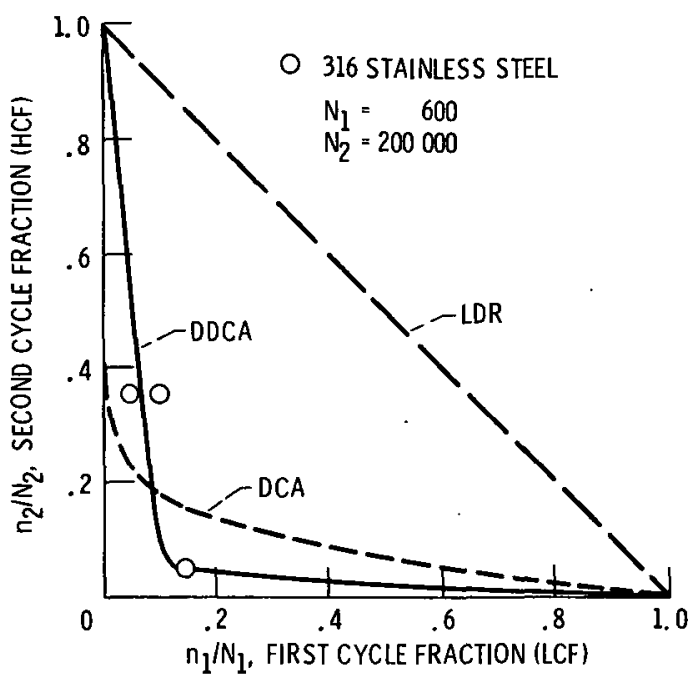

Fig. 25. Improved representation of HCF/LCF interactions using DDCA with optimized constants $(\alpha=0.23, \beta=0.63, \gamma=5.0)$. Comparison with LDR and $D C A(\beta=0.4)$.

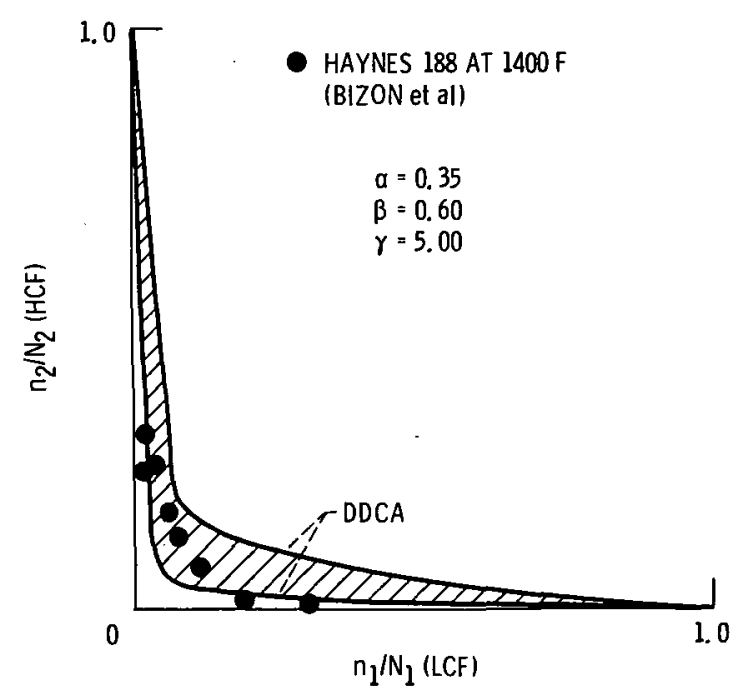

Fig. 26. Correlation of HCF/LCF interaction by the double curve approach (DDCA). Data from ref. (26). Cross-hatched area represents range of $\mathrm{N}_{1} / \mathrm{N}_{2}$

data. 


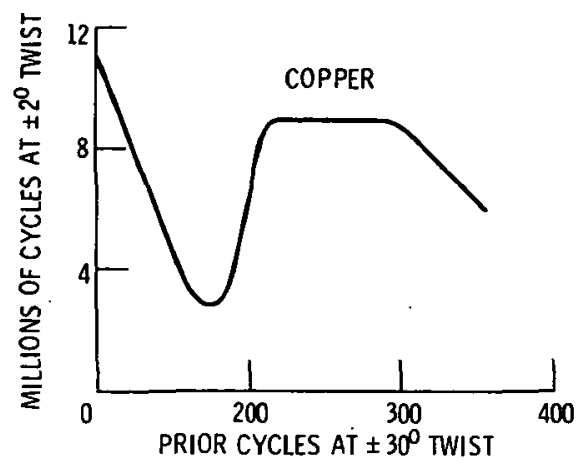

(a) Results for copper, after Wood and Reimann (31).

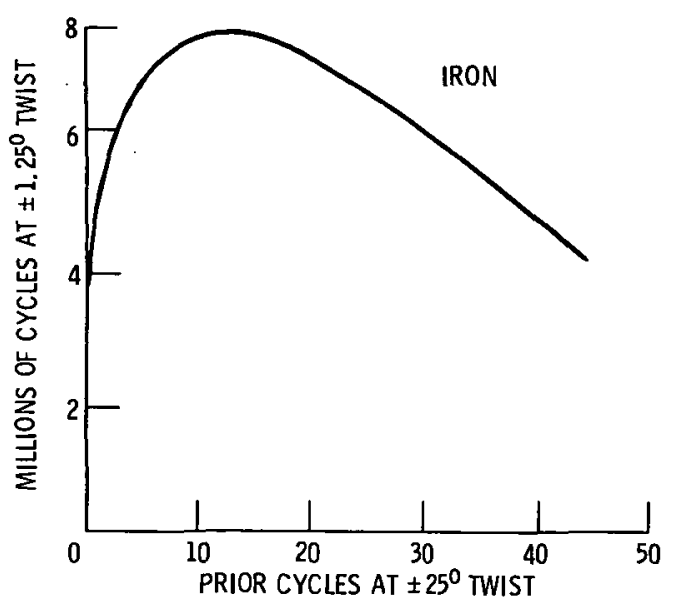

(b) Results for iron, after Reimann (32).

.Fig. 27. Unusual cumulative fatigue damage behavior in torsion.

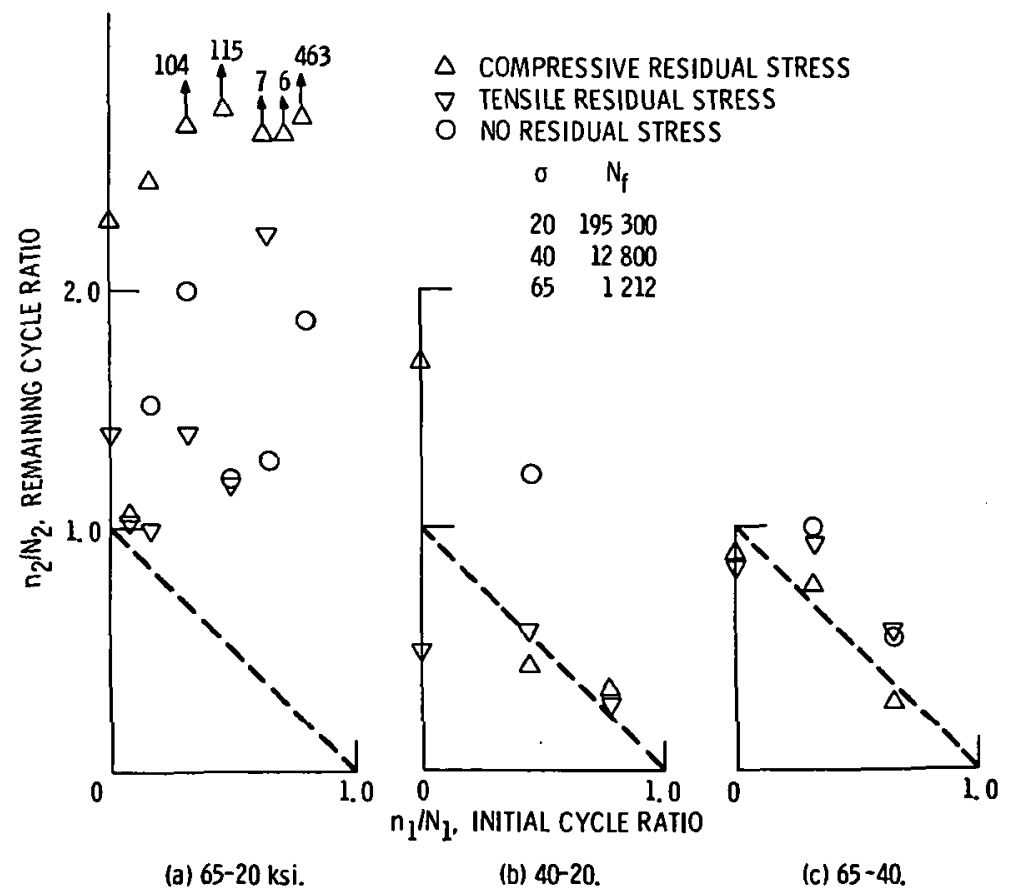

Fig. 28. Residual stress effects with 7075-T6 aluminum, ref. (33). 

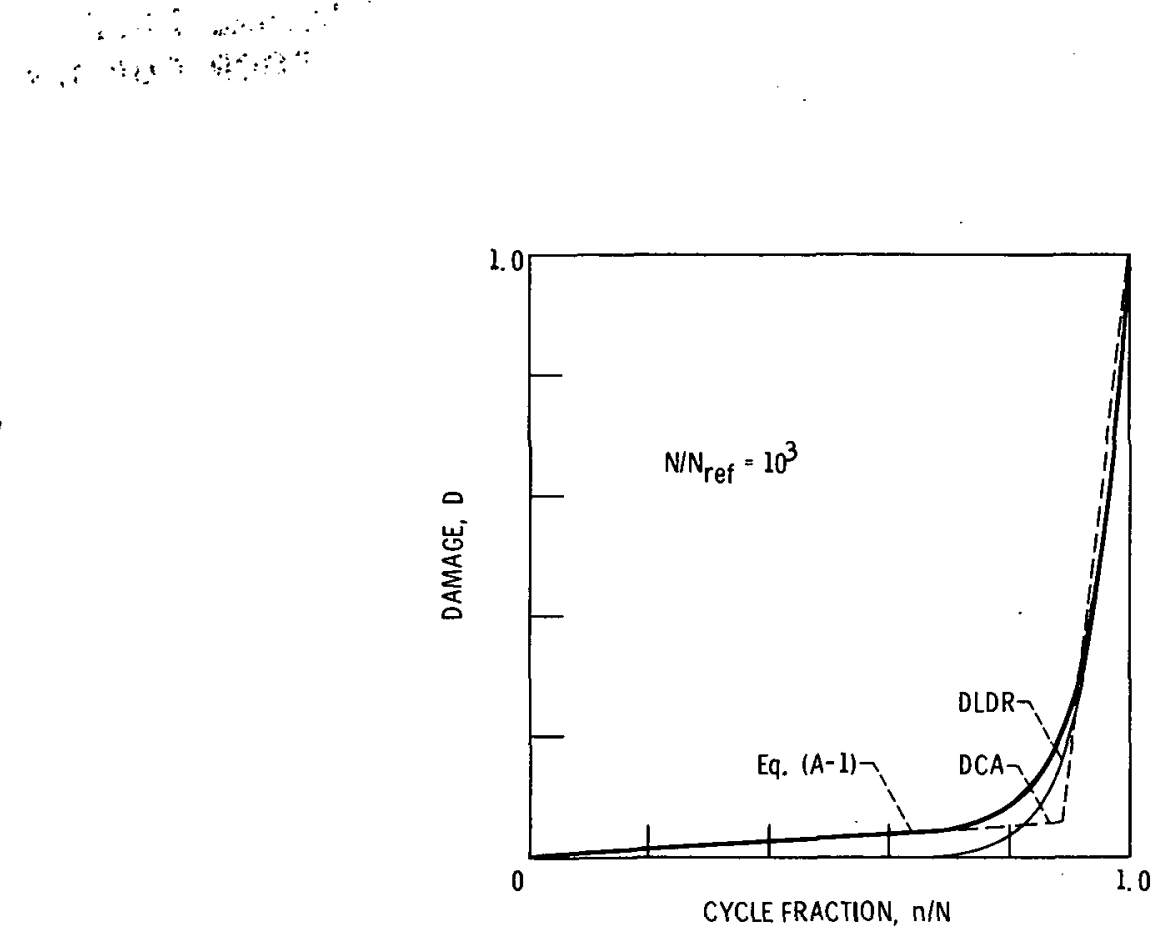

(a) Equation (A-1) conforms to DLDR at low values of cycle fraction.

Fig. A-1. Application of DLDR.

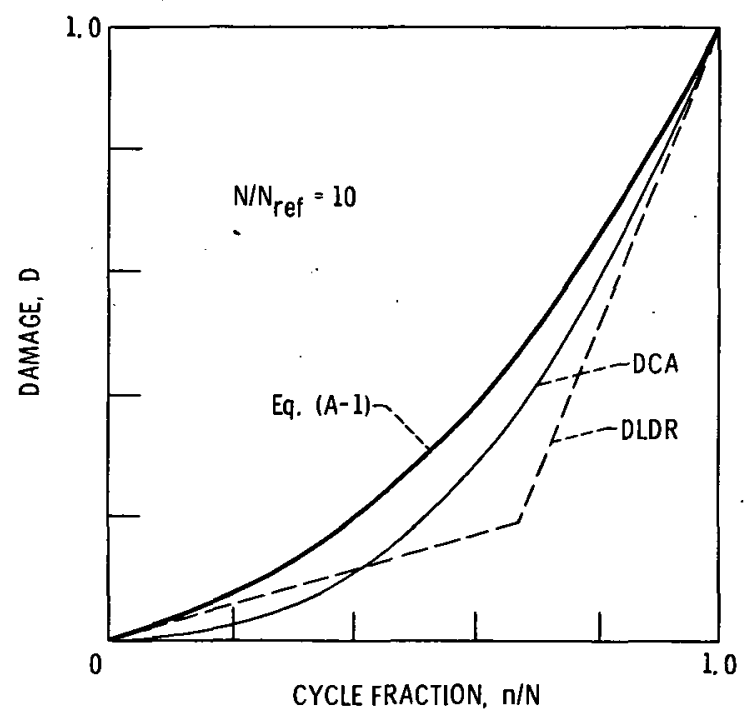

(b) Equation (A-1) does not conform well to DLDR at transition for $N / N_{\text {ref }}=10$.

Fig. A-1. Concluded. 


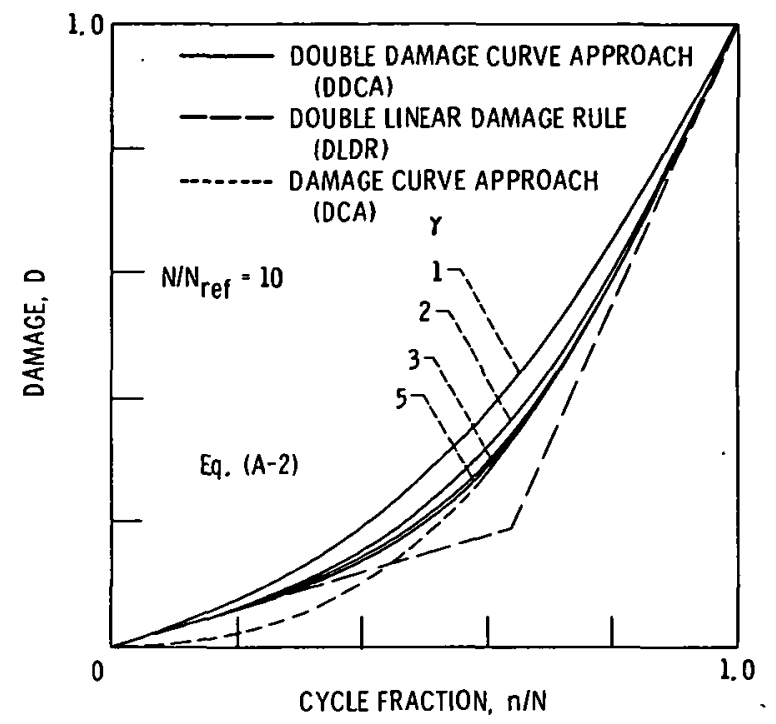

Fig. A-2. DDCA, Equation (A-2) follows DLDR for low values of damage and follows DCA for high values of damage for $\gamma=5$.

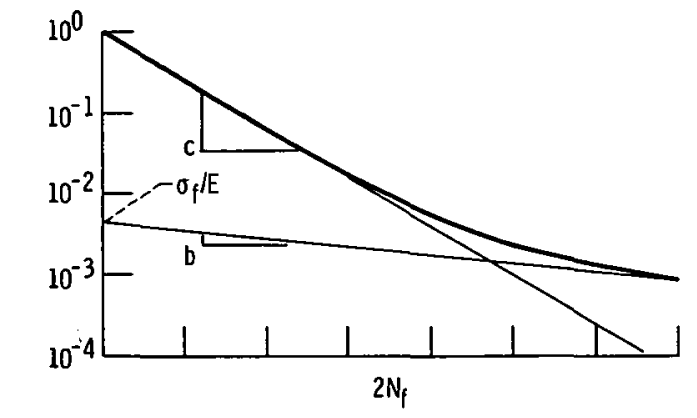

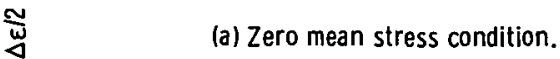

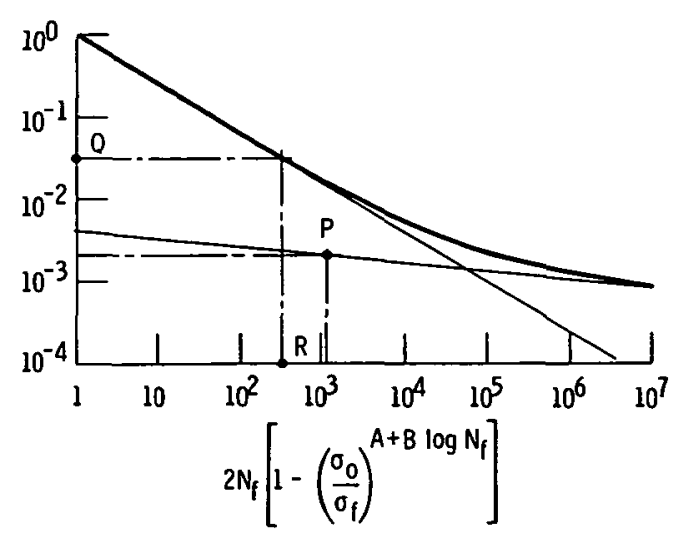

(b) General mean stress condition.

Fig. B-1. Manson-Coffin-Basquin fatigue relation. 


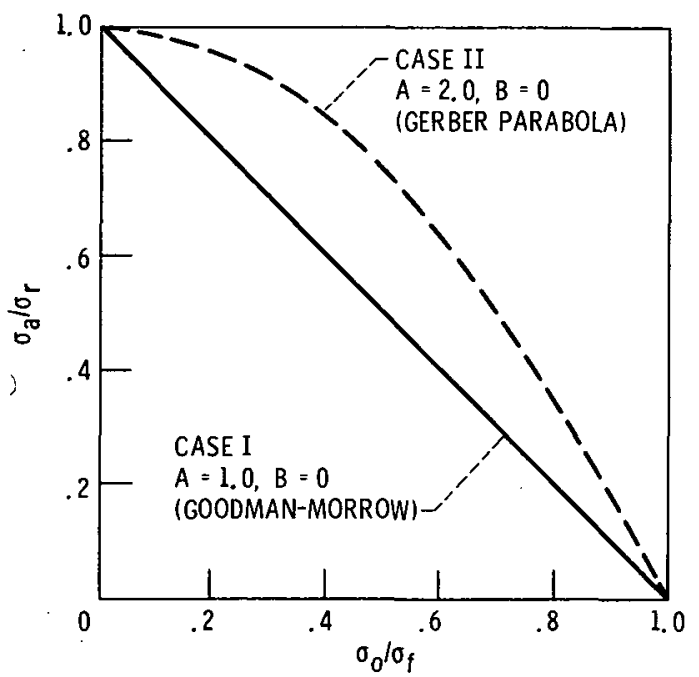

Fig. B-2. Normalized mean stress behavior for cases I and II. Valid for all life levels.

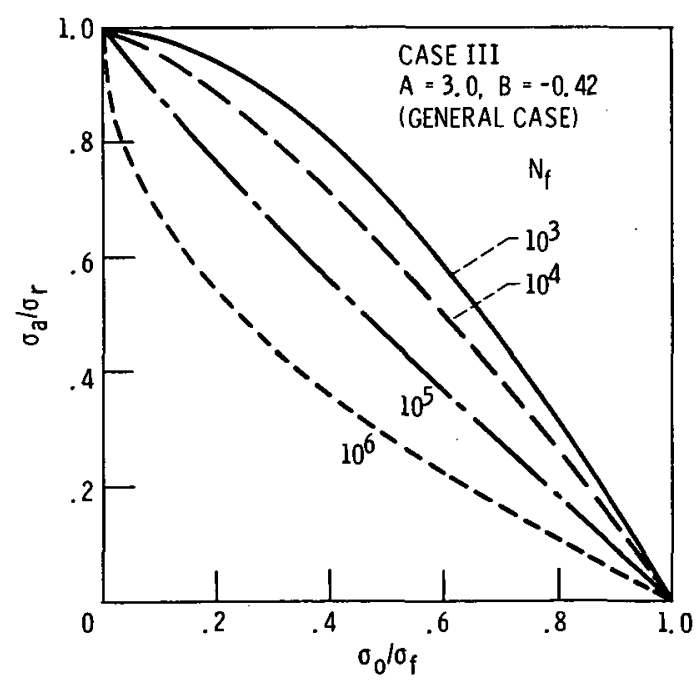

Fig. B-3. Normalized mean stress behavior for case III. Shape of curve changes with fatigue life level. 


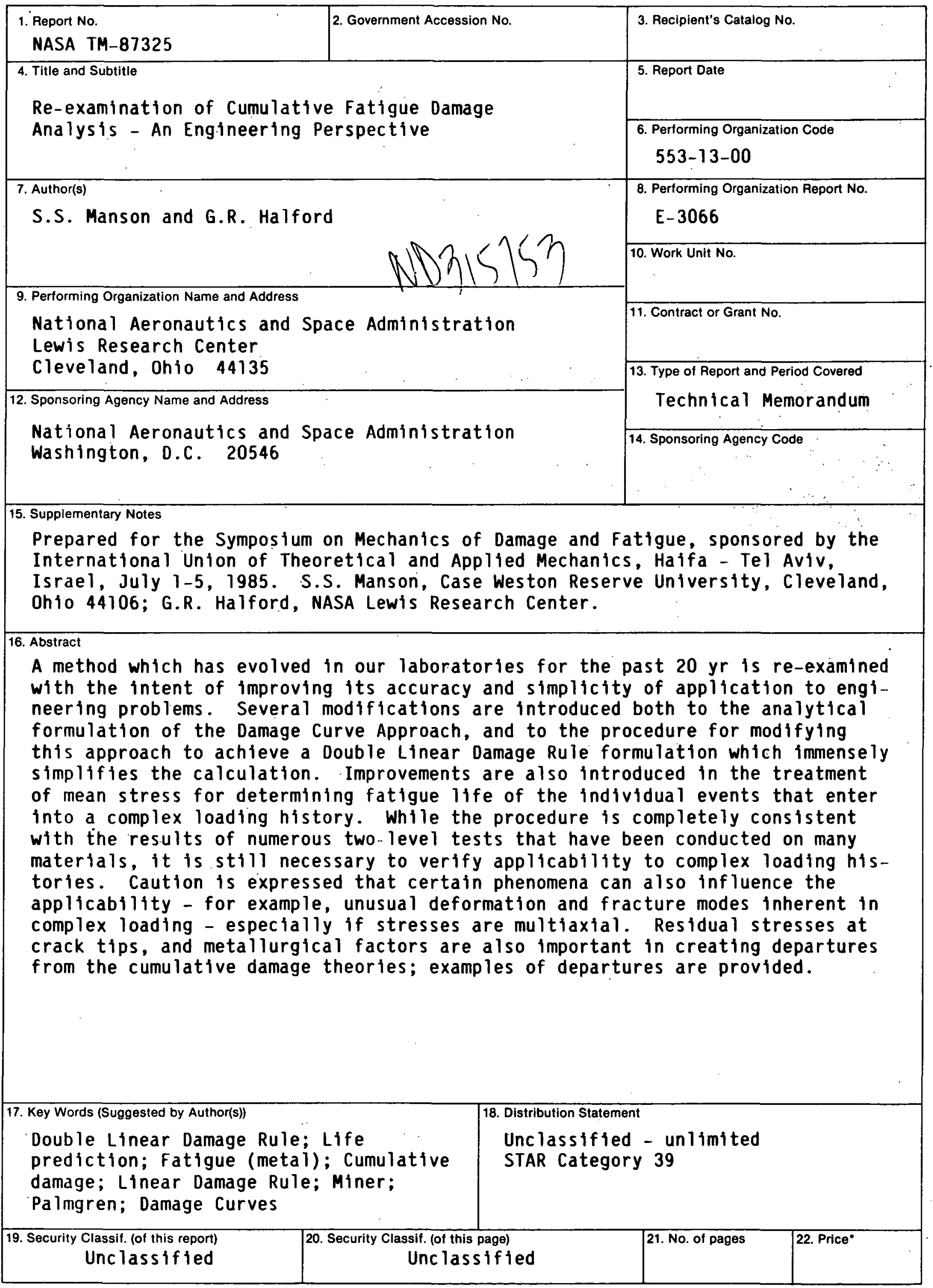


National Aeronautics and

Space Administration

Lewis Research Center

Cleveland. Ohio 44135

Official Business

Penalty for Private Use $\$ 300$

\section{SECOND CLASS MAIL}

ADDRESS CORRECTION REQUESTED

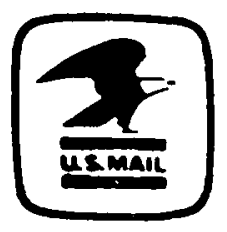

Postage and Fees Paid

National Aeronautics and

Space Administration

NASA-451 